\title{
A controlled human Schistosoma mansoni infection model to advance novel drugs, vaccines and diagnostics
}

\author{
Marijke C. C. Langenberg ${ }^{1}{ }^{1}$, Marie-Astrid Hoogerwerf ${ }^{1,7}$, Jan Pieter R. Koopman ${ }^{1}{ }^{1,7}$, \\ Jacqueline J. Janse', Janneke Kos-van Oosterhoud', Carola Feijt', Simon P. Jochems ${ }^{1}$ ', \\ Claudia J. de Dood², Roos van Schuijlenburg', Arifa Ozir-Fazalalikhan', Mikhael D. Manurung1, \\ Erliyani Sartono', Martha T. van der Beek³, Béatrice M. F. Winkel', Petra H. Verbeek-Menken", \\ Koen A. Stam', Fijs W. B. van Leeuwen ${ }^{5}$, Pauline Meij ${ }^{6}$, Angela van Diepen', Lisette van Lieshout', \\ Govert J. van Dam1, Paul L. A. M. Corstjens'2, Cornelis H. Hokke ${ }^{11,8}$, Maria Yazdanbakhsh ${ }^{1,8}$, \\ Leo G. Visser ${ }^{4,8}$ and Meta Roestenberg ${ }^{1,4}{ }^{1,}$
}

\begin{abstract}
Schistosomiasis treatment relies on the use of a single drug, praziquantel, which is insufficient to control transmission in highly endemic areas'. Novel medicines and vaccines are urgently needed ${ }^{2,3}$. An experimental human model for schistosomiasis could accelerate the development of these products. We performed a dose-escalating clinical safety trial in 17 volunteers with male Schistosoma mansoni cercariae, which do not produce eggs (clinicaltrials.gov NCT02755324), at the Leiden University Medical Center, the Netherlands. The primary endpoints were adverse events and infectivity. We found a dose-related increase in adverse events related to acute schistosomiasis syndrome, which occurred in 9 of 17 volunteers. Overall, 5 volunteers (all 3 of the high dose group and 2 of 11 of the medium dose group) reported severe adverse events. Worm-derived circulating anodic antigen, the biomarker of the primary infection endpoint, peaked in $82 \%$ of volunteers at 3-10 weeks following exposure. All volunteers showed IgM and IgG1 seroconversion and worm-specific cytokine production by $\mathrm{CD4}^{+} \mathrm{T}$ cells. All volunteers were cured with praziquantel provided at 12 weeks after exposure. Infection with 20 Schistosoma mansoni cercariae led to severe adverse events in $18 \%$ of volunteers and high infection rates. This infection model paves the way for fast-track product development for treatment and prevention of schistosomiasis.

Worldwide, 290 million people are infected with schistosomes, mainly Schistosoma haematobium and Schistosoma mansonit. The endemicity is determined by the presence of the fresh water snail intermediate host. Snail-derived cercariae penetrate the human skin and migrate into the vascular system, where mature male and female worms mate and produce $\sim 300$ eggs per day. S. mansoni eggs provoke inflammatory responses, which can lead to liver cirrhosis and portal hypertension ${ }^{5}$.
\end{abstract}

Current treatment and control of schistosomiasis relies on the use of a single drug, praziquantel. Mass drug administration with praziquantel does not protect from reinfection ${ }^{1}$ and thus provides insufficient control in highly endemic areas, creating the need for a vaccine ${ }^{2}$

Several schistosome antigens have been put forward as possible $S$. mansoni vaccine candidates, of which three are in clinical development: Sm-TSP-2, rSm14/GLA-SE and Sm-p80 ${ }^{6-8}$. These candidates aim for $>40 \%$ reduction in worm load for World Health Organization endorsement ${ }^{9}$, but higher levels are preferred ${ }^{10,11}$. To obtain efficacy data, large phase II and III field trials in Schistosomaendemic areas are needed ${ }^{12}$. In addition, increasing concerns of praziquantel resistance create a need for anti-schistosomal drug development ${ }^{3}$. Controlled human infection (CHI) trials can select drug and vaccine candidates early in clinical development and help prevent late clinical failure ${ }^{12}$. We thus aimed to develop a schistosome $\mathrm{CHI}$ model to aid vaccine and drug development and better characterize human anti-schistosome immune responses.

Between September 2016 and January 2018, 35 healthy adult volunteers were screened, of which 17 were included in the trial and completed follow up. (Fig. 1). Baseline demographics between dose groups were comparable (Supplementary Tables 1 and 2).

Volunteers were exposed to 10,20 or 30 cercariae in water on the forearm skin for $30 \mathrm{~min}$, after which they were followed bi-weekly for adverse events and infectivity. After exposure, water was pipetted off the skin and inspected for remaining cercariae. We found tails of roughly half the number of the cercariae that they were exposed to, with clear differences between exposure groups $(r=0.70, P=0.002$; Supplementary Table 1).

Safety. The majority of volunteers ( 15 of $17,88 \%)$ experienced pruritus during or after exposure, but no topical treatment was required

'Department of Parasitology, Leiden University Medical Center, Leiden, the Netherlands. 'Department of Cell and Chemical Biology, Leiden University Medical Center, Leiden, the Netherlands. ${ }^{3}$ Department of Medical Microbiology, Leiden University Medical Center, Leiden, the Netherlands. ${ }^{4}$ Department of Infectious Diseases, Leiden University Medical Center, Leiden, the Netherlands. ${ }^{5}$ Interventional Molecular Imaging Laboratory, Department of Radiology, Leiden University Medical Center, Leiden, the Netherlands. ${ }^{6}$ Department of Clinical Pharmacy and Toxicology, Leiden University Medical Center, Leiden, the Netherlands. ${ }^{7}$ These authors contributed equally: Marie-Astrid Hoogerwerf, Jan Pieter R. Koopman. ${ }^{8}$ These authors jointly supervised this work: Cornelis H. Hokke, Maria Yazdanbakhsh, Leo G. Visser. $\varpi_{e}$-mail: m.roestenberg@lumc.nl 


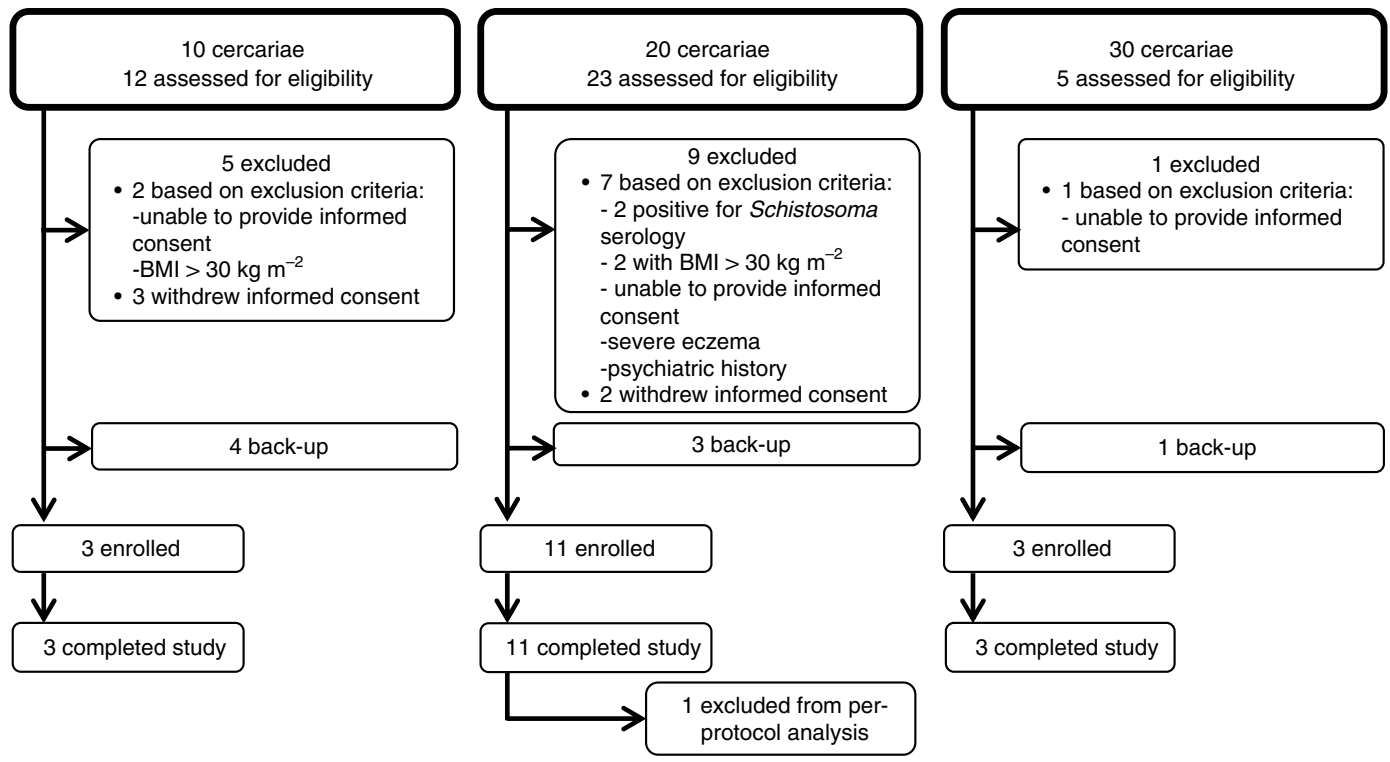

Fig. 1 Study flow diagram. The inclusion and exclusion of volunteers. The first group ( $n=3$ volunteers) was exposed to 10 cercariae and the second group ( $n=3$ volunteers) to 30 cercariae. Thereafter the dose was de-escalated to 20 cercariae for the third group $(n=3$ volunteers) and validated in a fourth group ( $n=8$ volunteers). In total eight volunteers served as backup for included volunteers in case an included volunteer might drop out before exposure to cercariae.

(Supplementary Table 3). All but one volunteer (16 of 17, 94\%) developed a mild local cercarial dermatitis within $2 \mathrm{~d}$ after exposure (Fig. 2a-c), which lasted longer in higher dose groups (10 cercariae: median 1 week (range 1-2); 20 cercariae: 3 weeks (1-9); 30 cercariae: 6 weeks $(2-7), r=0.45, P=0.07)$.

There were no serious adverse events (AEs) (an event that is life-threatening or requires hospitalization), but nine severe related AEs (resulting in the inability to perform daily activity) were reported in volunteers from the $20(n=2)$ or 30 cercariae $(n=3)$ group. Seven of these severe AEs were symptoms of an acute schistosomiasis syndrome $(n=5)$. In the 30 cercariae group all volunteers $(n=3)$ experienced severe AEs, starting 2.5 to 5.0 weeks after exposure (Fig. 2d-f) as follows: headache $(n=2)$, fever $(n=2)$, syncope $(n=1)$, nausea $(n=1)$ and elevated liver enzymes $(n=1)$ (Supplementary Table 3). In one volunteer this episode was followed by mild to moderate headaches, malaise, fatigue and nocturnal sweats for up to 6 weeks as previously described ${ }^{13}$, but steroid treatment was declined. Given the burden of these prolonged symptoms of an acute schistosomiasis syndrome (Katayama symptoms), defined as moderate to severe symptoms of malaise, fatigue, fever, night sweats, flu-like symptoms or headache, between 2 to 7 weeks after exposure, the dose was de-escalated to 20 cercariae. Subsequently, 2 of $11(18 \%)$ volunteers reported one severe symptom of an acute schistosomiasis syndrome (headache, nocturnal fever and sweats).

In addition to these five volunteers with severe AEs there were four volunteers, all exposed to 20 cercariae, with moderate symptoms of an acute schistosomiasis syndrome (flu-like symptoms, $n=3$; and malaise, $n=2$ ). Eight volunteers did not experience any symptoms of an acute schistosomiasis syndrome.

Eosinophils. Eosinophils increased in 11 of 17 (65\%) volunteers peaking between week 2 and 8 after infection $\left(0.1-8.4 \times 10^{9} 1^{-1}\right.$; Extended Data Fig. 1). Eosinophils were not related to the dose or symptoms (Extended Data Fig. 2a).

Infection rates by antigen detection assays. Both worm-excreted circulating anodic antigen (CAA) and circulating cathodic antigen (CCA) were measured to determine the presence and degree of infection. In two of three volunteers exposed to 10 cercariae the serum CAA was higher than $1 \mathrm{pg} \mathrm{ml}^{-1}$. In the higher dose groups, 9 out of 11 (82\%) volunteers exposed to 20 cercariae and all volunteers exposed to 30 cercariae crossed this threshold. The time to patency was comparable between these groups (range 3-8 weeks, Fig. 3a).

Cercarial dose and serum CAA levels were related (10 cercariae median at week $7-12$ : $0.4 \mathrm{pg} \mathrm{m}^{-1}$ (range $0.3-0.8$ ); 20 cercariae: $1.2 \mathrm{pg} \mathrm{ml}^{-1}(0.3-1.9)$; and 30 cercariae: $3.6 \mathrm{pg} \mathrm{ml}^{-1}(2.0-6.5), r=0.70$, $P=0.002$ ) (Fig. 3b). The volunteer experiencing the most severe AEs had at least sevenfold higher serum CAA levels (maximum $49.9 \mathrm{pg} \mathrm{ml}^{-1}$ ) than other volunteers. There was no significant correlation between serum CAA levels and symptoms of an acute schistosomiasis syndrome (Extended Data Fig. 2b) or eosinophils (data not shown).

Urine CAA levels were variable (Extended Data Fig. 3a,b), but correlated with serum CAA $(r=0.58, P<0.0001)$. The point-of-care rapid test for CCA (POC-CCA) was positive in 12\% (2 of 17) of volunteers within 12 weeks after exposure and did not correlate with serum or urine CAA levels.

Praziquantel treatment. After a single $40 \mathrm{mg} \mathrm{kg}^{-1}$ dose of praziquantel treatment 12 weeks after exposure, serum CAA levels dropped below the detection limit in 8 out of 14 (57\%) positive volunteers. The remaining $43 \%$ of volunteers were treated again with praziquantel, after which all remained undetectable until 1 year after exposure (Extended Data Fig. 4a-c).

Serology. All volunteers showed seroconversion of IgM against adult worms by immunofluorescence assay (IFA) (Fig. 4a,b). Seroconversion became apparent at week 4 in two volunteers and by week 6 in all. In addition, seven volunteers showed anti-soluble egg antigen (SEA) IgG seroconversion respectively at week 4 ( 1 of 16), week 12 ( 1 of 16 ) or week 20 (5 of 16). The absence of eggs was confirmed by a negative feces Schistosoma PCR at week 12 in all volunteers.

All volunteers showed an increase in adult worm antigen (AWA)specific total IgG over time, with seroconversion above $2 \times$ s.d. of all 


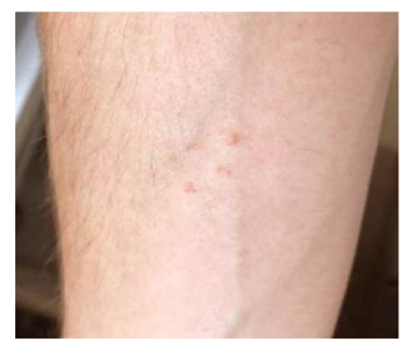

d

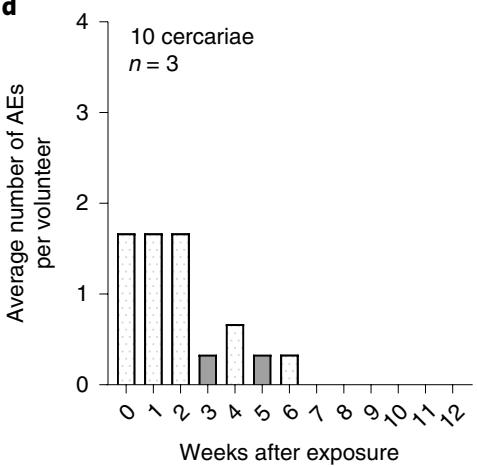

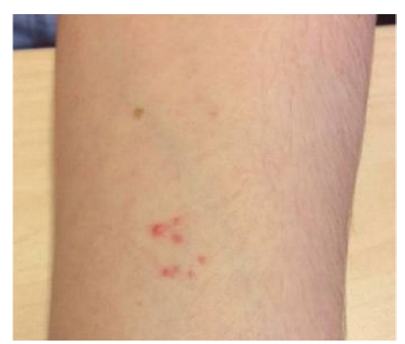

e

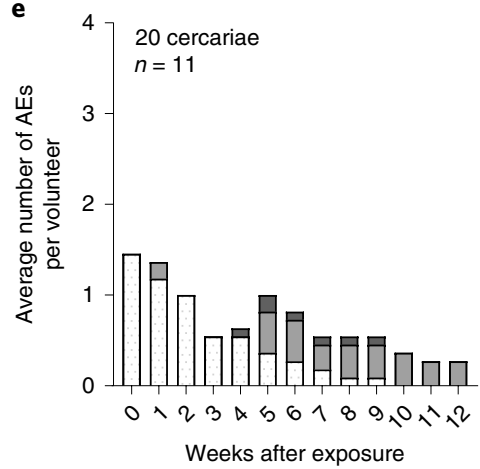

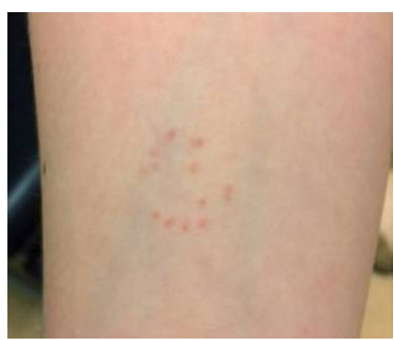

$\mathbf{f}$

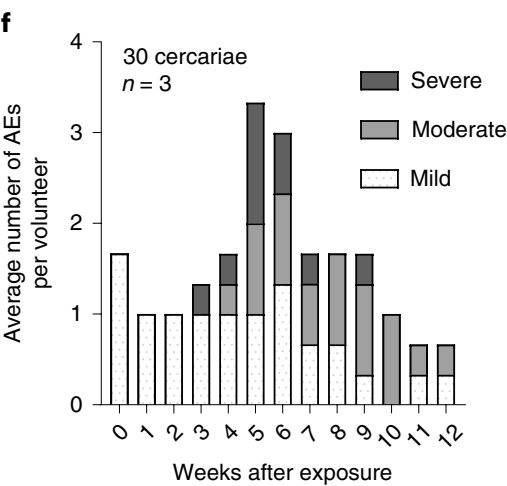

Fig. 2 | Adverse events. a-c, Pictures of volunteers with the most pronounced cercarial dermatitis taken $5 \mathrm{~d}$ after infection from the group infected with 10 cercariae (a), 20 cercariae (b) or 30 cercariae (c). d-f, The average number of related AEs per volunteer at each week for volunteers exposed to 10 cercariae (d), 20 cercariae (e) or 30 cercariae (f). For each AE the highest grading score is plotted. White bar, mild AE; light gray bar, moderate AE; dark gray bar, severe $A E$.

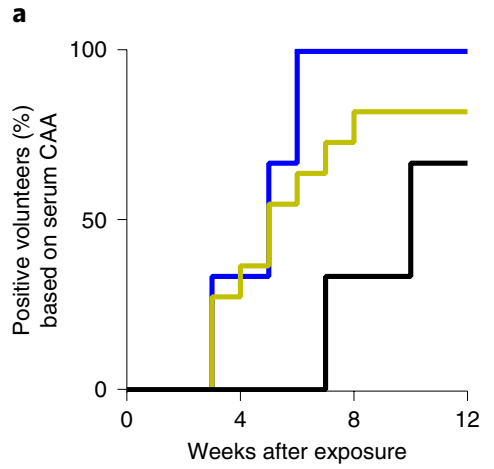

-10 cercariae
-20 cercariae
-30 cercariae

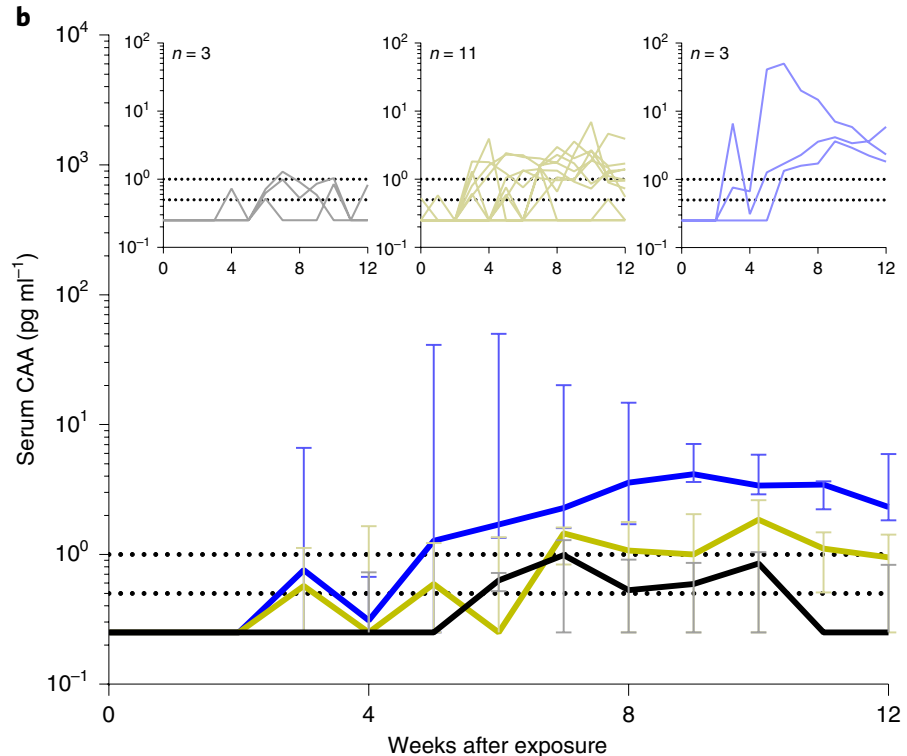

Fig. 3 | Pre-patent period and serum CAA levels. a, The time to patency (serum CAA level $>1$ pg ml ${ }^{-1}$ ) in weeks per dose group (log-rank test, $P=0.21$ ). b. The serum CAA levels for individual volunteers in $\mathrm{pg} \mathrm{m}^{-1}$ (top) and the median serum CAA levels and interquartile range for groups infected with 10,20 or 30 cercariae (bottom). The gray, yellow and blue lines represent data of individual volunteers or groups exposed to 10 ( $n=3$ ), 20 ( $n=11)$ or $30(n=3)$ cercariae respectively.

baseline values in 12 of 16 volunteers at week 20. These responses were primarily IgG1, with seroconversion in all volunteers by week 16 (Fig. 4c,d). There was a clear dose response in AWA-specific total IgG and IgG1 levels (IgG week 20: $r=0.70, P=0.003$; IgG1 week 16: $r=0.56, P=0.02)$, and a trend toward higher IgG1 levels and symptoms of an acute schistosomiasis syndrome $(P=0.08)$ (Extended
Data Fig. 2c). No changes over time were found in total IgE or AWAspecific IgE and IgG4 compared to baseline (data not shown).

Cytokines. Cytokine and chemokine measurements in serum revealed increases in innate chemokines interferon (IFN)- $\gamma$ inducible protein (IP)-10 (Fig. 5a,b) and macrophage inflammatory 


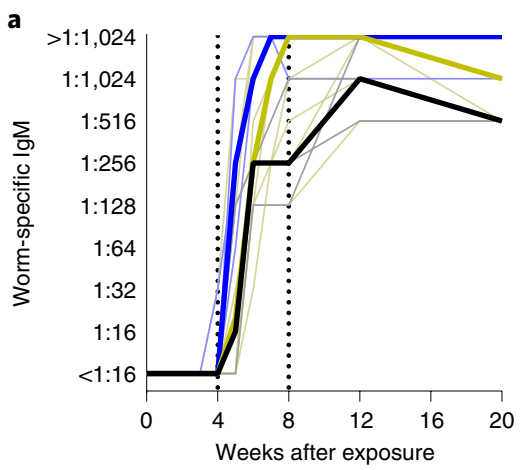

b

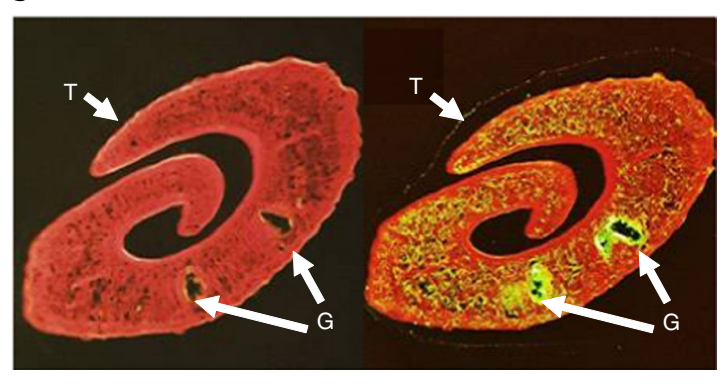

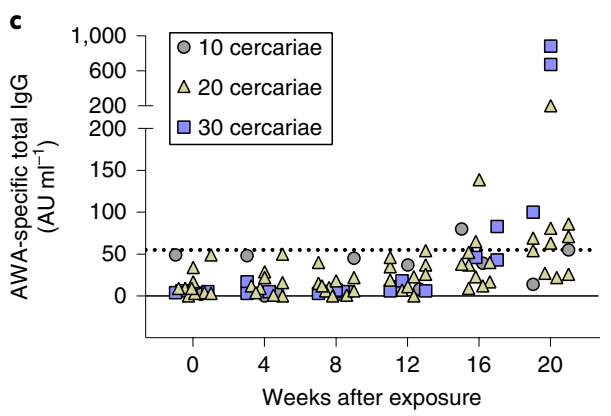

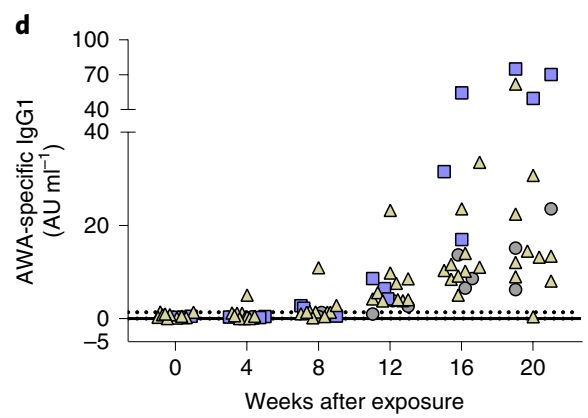

Fig. 4 | Humoral immune response. a, IgM against adult worm antigens over time. The gray, yellow and blue lines represent data of individual volunteers exposed to $10(n=3), 20(n=10)$ or $30(n=3)$ cercariae respectively and the darker lines represent the median of each group. $\mathbf{b}$, IFA showing reactivity of volunteer IgM antibodies to Rossman-fixed S. mansoni adult worm sections (right) and pre-exposure negative serum (left). T, tegument; G, gastrodermis. c, AWA-specific total lgG for individual volunteers over time. d, AWA-specific lgG1 for individual volunteers over time. The gray circles, yellow triangles and blue squares represent data of individual volunteers exposed to $10(n=3), 20(n=10)$ or $30(n=3)$ cercariae respectively.

protein (MIP)-1 $\beta$ (Fig. 5c), the latter of which was significantly higher in volunteers with symptoms of an acute schistosomiasis syndrome $(P=0.01$ at week 8 , Fig. $5 \mathrm{~d})$. There were no detectable changes in the other circulating chemokines or cytokines measured in serum (data not shown).

Cellular immunology. Overall, the frequency of antigen-specific IFN- $\gamma(P=0.01)$ and Th2 cytokine (interleukin (IL)-4, IL-5 and IL-13, $P=0.004)$-producing $\mathrm{CD}^{+}{ }^{+} \mathrm{T}$ cells increased over time (Fig. 5e and Extended Data Fig. 5a,b), but FOXP $3^{+} \mathrm{CD} 25^{+} \mathrm{CD} 4^{+}$regulatory T cells $(P=0.49)$ or the production of the regulatory cytokine IL-10 $(P=0.91$, Fig. $5 \mathrm{e})$ did not. However, in volunteers with symptoms of an acute schistosomiasis syndrome, both IFN- $\gamma$ and Th2 cytokine-producing $\mathrm{CD}^{+} \mathrm{T}$ cells and $\mathrm{CD} 25^{+} \mathrm{FOXP} 3^{+}$regulatory $\mathrm{T}$ cells were higher at week 4 and week 8 respectively, although not significantly for all $(P=0.28, P=0.01$ and $P=0.02$, respectively; Fig. 5f). There were no differences in IL-10-cytokine-producing $\mathrm{CD}^{+} \mathrm{T}$ cells at week 4 (Fig. 5f). At all timepoints $17 \%$ of IFN$\gamma^{+} \mathrm{CD} 4^{+} \mathrm{T}$ cells also produced IL-2.

Data integration. To understand which immunological and microbiological datasets were associated with the occurrence of symptoms of an acute schistosomiasis syndrome, we performed data integration using parallel generalized canonical correlation and partial least squares discriminant analysis. Eosinophil data decreased model accuracy and were thus removed (Extended Data Fig. 6a,b). At baseline, 9 of $16(56.3 \%)$ participants were accurately predicted by the model with leave-one-out cross-validation, which increased to 13 of $16(81.3 \%)$ correct classifications at week 12 and further to 15 of $16(93.8 \%)$ when data from all timepoints were included (Fig. 6a,b). Thus, the model was able to accurately classify participants by the presence of an acute schistosomiasis syndrome. Permutation analysis confirmed that symptoms were strongly associated with the measured immunological and microbiological parameters over the infection course $(n=1,000$; 99.6 th percentile, Extended Data Fig. 6c). We identified Th2 cytokines at week 4 and FOXP3 regulatory T cells at weeks 12 and 16, MIP-1 $\beta$ at weeks 8 and 12 and levels of serum CAA at week 9 as important features elevated in symptomatic participants by leave-one-out cross-validation (Fig. 6c). Levels of Th2 cytokines upon stimulation at week 4 correlated with serum CAA at week 9, whereas concentrations of MIP-1 $\beta$ in plasma correlated with FOXP3 regulatory T cell numbers (Extended Data Fig. 6d).

Discussion. This pilot study shows that experimental exposure to 20 male cercariae results in a detectable and well-tolerated S. mansoni infection in $82 \%$ of volunteers. This infection rate resembles that of other human infection models $\mathrm{s}^{14}$. All volunteers were cured after 1-2 doses of praziquantel.

The dose-escalating design of the study revealed a concomitant increase in AEs. The occurrence of a severe acute schistosomiasis (Katayama) syndrome in one volunteer prompted us to lower the dose. At a dose of 20 cercariae, 2 of 11 volunteers reported severe AEs, which is comparable with other human infection models. For example, in experimental malaria infections, volunteers generally experience three to four AEs, of which one would be severe for several days ${ }^{15}$. In typhoid infections $\sim 50 \%$ of volunteers report severe symptoms and $55 \%$ experiences fever ${ }^{16}$ and in cholera infection studies $40 \%$ of volunteers experience 1.6-8.0 liters of diarrhea ${ }^{17}$. We thus conclude that 20 cercariae may be the optimal dose that was both well tolerated and detectable. A relationship between dose and symptoms, as suggested by epidemiological data ${ }^{18}$, cannot be statistically confirmed with the current number of volunteers. Regardless of dose, all symptoms had resolved without sequelae at 12 weeks.

The follow up of volunteers in our study unequivocally showed that anti-adult worm $\operatorname{IgM}(100 \%$ seroconversion by week 6$)$ or serum CAA (10 of 17 positive by week 6) are the earliest diagnostic markers currently available. This is in accordance with previous 

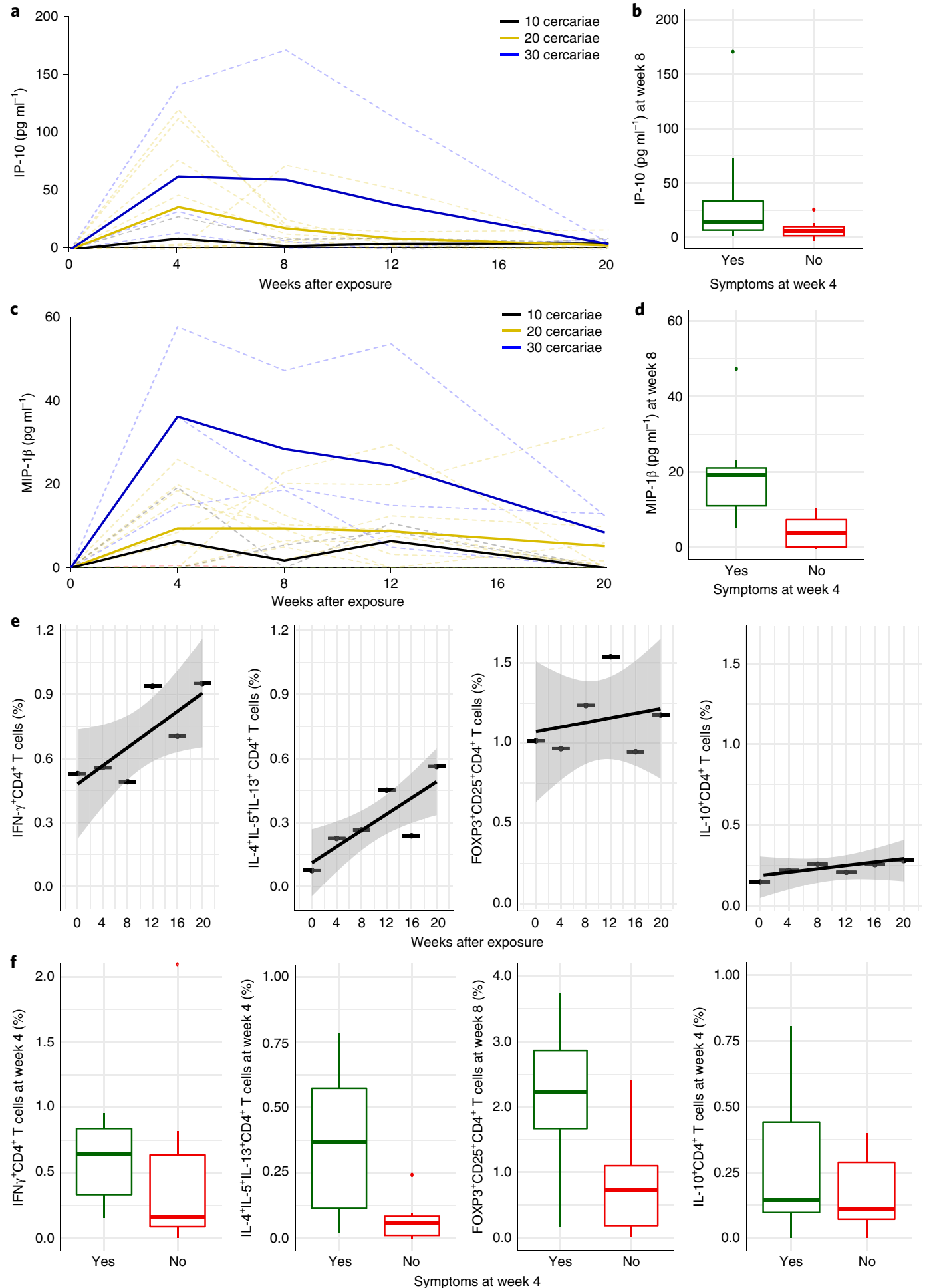

Fig. 5 | Cytokine responses. a, Ex vivo IP-10 over time for individual volunteers (dotted line) and the mean of each group (line). b, IP-10 levels at week 8 in volunteers with (yes, $n=8$ ) or without (no, $n=8$ ) symptoms of an acute schistosomiasis syndrome (two-sided Mann-Whitney $U$-test, $P=0.16$ ). c, Ex vivo MIP-1 $\beta$ over time for individual volunteers (dotted line) and the mean of each group (line). d, MIP-1 $\beta$ levels at week 8 in volunteers with (yes, $n=8$ ) or without (no, $n=8$ ) symptoms of an acute schistosomiasis syndrome at week 4 (two-sided Mann-Whitney $U$-test, $P=0.01$ ). e, The percentage of IFN- $\gamma$-producing CD4 ${ }^{+}$T cells $(P=0.01)$, Th2 cytokine (IL-4, IL-5 and IL-13)-producing CD4+ ${ }^{+}$cells $(P=0.004), F O X P 3^{+} C D 25^{+} C D 44^{+} T$ cells $(P=0.49)$ and IL-10-producing CD4 ${ }^{+}$T cells $(P=0.91)$ over time for all volunteers $(n=16)$, showing average of all volunteers at each week and fitted linear regression line with $95 \%$ confidence interval (linear mixed model with two-sided Student's t-test, using the Satterthwaite's degrees of freedom method). f, The percentage of IFN- $\gamma$-producing CD4+ $T$ cells $(P=0.28)$, Th2 cytokine $\left(I L-4, I L-5\right.$ and IL-13)-producing CD4 ${ }^{+}$T cells $(P=0.01)$, FOXP3 ${ }^{+} C D 25^{+} C D 4^{+}$ T cells $(P=0.02)$ and IL-10-producing CD4 ${ }^{+}$T cells $(0.56)$ in volunteers with (yes, $\left.n=8\right)$ or without $(n o, n=8)$ symptoms of an acute schistosomiasis syndrome. In all boxplots, the lower and higher hinge are respectively the first and third quartiles and the middle line is the median (second quartile). The whiskers extend from the hinge to the largest value no further than $1.5 \times$ the interquartile range or distance between the first and third quartile. The largest and lowest visible points beyond the whiskers extend this range and can then be considered, respectively, the maxima and minima. 

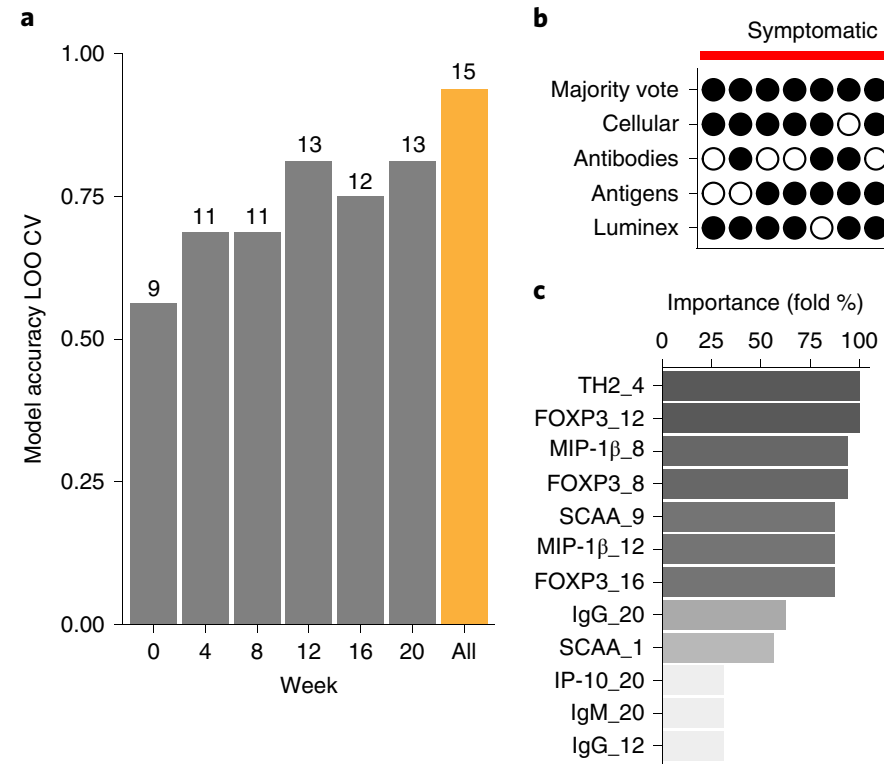

Asymptomatic
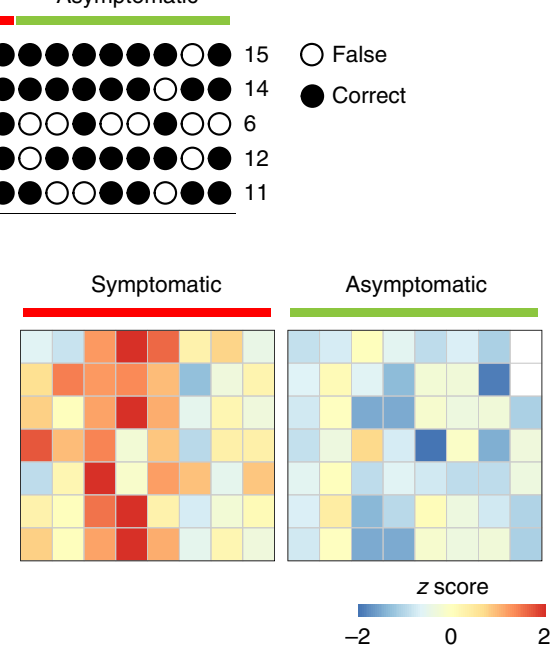

Fig. 6 | Immunological and microbiological data integration. Cellular, antibody, antigen and Luminex data were combined with generalized canonical correlation analysis and simultaneously associated with presence of symptoms through discriminant analysis. a, Proportion of correct predictions of each model assessed using leave-one-out cross-validation. Gray bars indicate data from one specific timepoint and orange bars indicate the model, including all data across timepoints. The number of correct predictions is indicated above each bar. b, Individual predictions across folds for the full model per dataset, including majority vote, with weighing to break ties. Each symbol represents one prediction, with volunteers in columns and datasets in rows. Filled circles indicate a correct prediction and open circles a false prediction. Volunteers with (red, $n=8$ ) or without (green, $n=8$ ) symptoms of acute schistosomiasis syndrome are indicated, as are the number of correct predictions per dataset. consensus features selected in at least $25 \%$ of the folds are indicated and ranked by frequency of presence among folds. The number after each feature indicates the week of measurement. The $z$ score-normalized levels of the seven features present in at least $75 \%$ of the folds are indicated in the heat map, where each column corresponds to one volunteer. Missing data points from one asymptomatic participant are depicted in white.

studies in travelers ${ }^{19-21}$. We found levels of serum CAA comparable to those in low endemic settings ${ }^{22}$. In nonhuman primate models these levels reflect 5-24 cercariae reaching adulthood ${ }^{8,23}$. Although serum CAA levels were more stable compared to urine CAA, the higher volume input of urine allows for more sensitive detection ${ }^{24}$. As suggested in previous studies, the urine POC-CCA rapid test was not suitable to detect very low intensity infection ${ }^{25}$. The main advantage of measuring serum CAA levels, as opposed to antibody detection, is the potential to follow up after treatment and confirm cure. In doing so, we found low cure rates with a single dose of $40 \mathrm{mg} \mathrm{kg}^{-1}$ praziquantel. Although a $60 \mathrm{mg} \mathrm{kg}^{-1}$ dose seemed more effective, the subsequent changes in pharmacokinetic and pharmacodynamic parameters need to be evaluated to conclude this with certainty. Because of the synchronous development of adult worms and the absence of reinfection in our model, the CHI design allows for screening of existing and new (stage-specific) anthelmintics.

The controlled schistosome infection model presented here clearly differs from infections in endemic settings, where doses are not controlled and infection occurs repeatedly. In addition, the single-sex infection lacks production of eggs that drive chronic regulatory and modified Th2 responses ${ }^{26}$. The presence of IgG to soluble egg antigen, indicates cross-reactive epitopes between eggs and worms. The induction of chemokines IP-10 and MIP-1 $\beta$ aligned with the increase in both antigen-specific CD4 ${ }^{+}$T cell IFN- $\gamma$ and Th2 cytokine production, respectively. The IFN- $\gamma$ production contrasts with predominantly Th2 profiles in epidemiological studies, which may be driven by egg-related responses ${ }^{26}$. Although we detected increases in $\mathrm{FOXP}^{+} \mathrm{CD} 25^{+} \mathrm{CD} 4^{+} \mathrm{T}$ cells, these mainly occurred in symptomatic volunteers and were found at a later timepoint than IFN- $\gamma$ - or Th 2 cytokine-producing T cells. This would be in line with the induction of regulatory $\mathrm{T}$ cells to prevent deleterious immune responses ${ }^{27}$, different from the chronic regulation found in endemic infections. These important differences in antigen exposure and subsequent immunological responses between single-sex infections and endemic chronic egg production may limit the use of the model to study anti-fecundity effects. However, the immunological observations from our model are comparable to acute infection models in travelers and baboons, where a mixed Th1 and Th2 response also dominates ${ }^{28,29}$. Integrated data analysis revealed that acute schistosomiasis symptoms were particularly associated with antigen-specific Th2 cytokine production and circulating MIP-1 $\beta$ and IgM production, but could not be predicted at baseline. In contrast to volunteers with symptoms, those without symptoms predominantly showed IgG1 antibody responses. Similarly to what has been performed for other infectious diseases ${ }^{30}$, a trial with repeated controlled infections could address whether these IgM or IgG1 antibodies have a protective effect. Currently, the clinical and immunological data from baseline alone cannot predict who will become symptomatic. However, the analysis of longitudinal responses reveals a clear profile predicting presence or absence of symptoms in 15 out of 16 participants. Unraveling the interplay between symptoms, immune responses and resistance to schistosome reinfection provides an opportunity for identification of new antigens for vaccine development ${ }^{31}$.

Immunological models for protection against S. mansoni were developed in rodents and nonhuman primates by repeated exposure to radiation attenuated cercariae ${ }^{23,32}$. In humans, three candidate $S$. mansoni vaccines are currently in clinical development ${ }^{6}$. A phase I study with rSm14/GLA-SE showed a good safety profile and immunogenicity ${ }^{7}$. Phase I safety results for Sm-TSP-2 are expected soon (ClinicalTrials.gov identifier: NCT02337855), while Sm-p80 is currently entering phase I testing on the basis of promising efficacy data in baboons ${ }^{8}$. The limited funding opportunities and large sample size required for phase III testing preclude 
testing of all three candidates in phase III trials. Despite the differences between chronic $S$. mansoni infection in the field and the controlled human S. mansoni infection, the model provides an opportunity to obtain preliminary efficacy data on these vaccines and reduce the costs by allowing selection of the most promising candidates, which may be co-formulated to maximize benefit ${ }^{12}$.

Future vaccine studies aim at a $75 \%$ infection reduction in worm burden and egg output ${ }^{10}$. As there is a clear relationship between worm burden and serum CAA levels ${ }^{24,33}$, we propose that the reduction in worm burden can be determined by measuring the median serum CAA level between week 7-12. Assuming an $80 \%$ power and $\alpha=0.05$, this would require a group size of 11 individuals per arm. Currently the main limitation of our model is the use of male schistosomes only. Consequently vaccine targets that are more commonly expressed on females, such as the Sm-p80 vaccine candidate ${ }^{8}$, cannot be fully evaluated. A female worm infection model would be of value to dissect mechanisms of action and sex-specificity of vaccines.

We conclude that this controlled human S. mansoni infection model results in an $82 \%$ infection rate with few severe side effects at a dose of 20 cercariae. In addition, this model provides insight into the onset of symptoms of a schistosome infection, the ensuing immune response and the performance of diagnostic tests over time. Notably, this model paves the way for cost-effective and rapid proof-of-concept testing of new vaccines and drugs.

\section{Online content}

Any methods, additional references, Nature Research reporting summaries, source data, extended data, supplementary information, acknowledgements, peer review information; details of author contributions and competing interests; and statements of data and code availability are available at https://doi.org/10.1038/s41591020-0759-x.

Received: 6 June 2019; Accepted: 9 January 2020;

Published online: 17 February 2020

\section{References}

1. King, C. H. et al. Utility of repeated praziquantel dosing in the treatment of schistosomiasis in high-risk communities in Africa: a systematic review. PLoS Negl. Trop. Dis. 5, e1321 (2011).

2. Tukahebwa, E. M., Vennervald, B. J., Nuwaha, F., Kabatereine, N. B. \& Magnussen, P. Comparative efficacy of one versus two doses of praziquantel on cure rate of Schistosoma mansoni infection and re-infection in Mayuge District, Uganda. Trans. R. Soc. Trop. Med. Hyg. 107, 397-404 (2013).

3. Bergquist, R., Utzinger, J. \& Keiser, J. Controlling schistosomiasis with praziquantel: how much longer without a viable alternative? Infect. Dis. Poverty 6, 74 (2017).

4. Global Burden of Disease Study 2013 Collaborators. Global, regional, and national incidence, prevalence, and years lived with disability for 301 acute and chronic diseases and injuries in 188 countries, 1990-2013: a systematic analysis for the Global Burden of Disease Study 2013. Lancet 386, 743-800 (2015).

5. Colley, D. G., Bustinduy, A. L., Secor, W. E. \& King, C. H. Human schistosomiasis. Lancet 383, 2253-2264 (2014).

6. Merrifield, M. et al. Advancing a vaccine to prevent human schistosomiasis. Vaccine 34, 2988-2991 (2016).

7. Santini-Oliveira, M. et al. Schistosomiasis vaccine candidate Sm14/GLA-SE: phase 1 safety and immunogenicity clinical trial in healthy male adults. Vaccine 34, 586-594 (2016).

8. Zhang, W. et al. Sm-p80-based schistosomiasis vaccine: double-blind preclinical trial in baboons demonstrates comprehensive prophylactic and parasite transmission-blocking efficacy. Ann. NY Acad. Sci. 1425, 38-51 (2018).

9. Status of Vaccine Research and Development of Vaccines for Schistosomiasis (World Health Organization, 2014); http://who.int/immunization/research/ meetings_workshops/Schistosomiasis_VaccineRD_Sept2014.pdf
10. Mo, A. X. \& Colley, D. G. Workshop report: schistosomiasis vaccine clinical development and product characteristics. Vaccine 34, 995-1001 (2016).

11. Siddiqui, A. J. et al. Sm-p80-based vaccine trial in baboons: efficacy when mimicking natural conditions of chronic disease, praziquantel therapy, immunization, and Schistosoma mansoni re-encounter. Ann. NY Acad. Sci. 1425, 19-37 (2018).

12. Roestenberg, M., Hoogerwerf, M. A., Ferreira, D. M., Mordmuller, B. \& Yazdanbakhsh, M. Experimental infection of human volunteers. Lancet Infect. Dis. 18, e312-e322 (2018).

13. Langenberg, M. C. C. et al. Katayama syndrome without Schistosoma mansoni eggs. Ann. Intern. Med. 170, 732-733 (2019).

14. Balasingam, S. \& Wilder-Smith, A. Randomized controlled trials for influenza drugs and vaccines: a review of controlled human infection studies. Int. J. Infect. Dis. 49, 18-29 (2016).

15. Walk, J. et al. Diagnosis and treatment based on quantitative PCR after controlled human malaria infection. Malar. J. 15, 398 (2016).

16. Jin, C. et al. Efficacy and immunogenicity of a Vi-tetanus toxoid conjugate vaccine in the prevention of typhoid fever using a controlled human infection model of Salmonella typhi: a randomised controlled, phase 2b trial. Lancet 390, 2472-2480 (2017).

17. Chen, W. H. et al. Single-dose live oral cholera vaccine CVD 103-HgR protects against human experimental infection with Vibrio cholerae O1 El Tor. Clin. Infect. Dis. 62, 1329-1335 (2016).

18. Rocha, M. O. et al. Pathogenetic factors of acute Schistosomia mansoni: correlation of worm burden, IgE, blood eosinophilia and intensity of clinical manifestations. Trop. Med. Int. Health 1, 213-220 (1996).

19. van Grootveld, R. et al. Improved diagnosis of active Schistosoma infection in travellers and migrants using the ultra-sensitive in-house lateral flow test for detection of circulating anodic antigen (CAA) in serum. Eur. J. Clin. Microbiol. Infect. Dis. 37, 1709-1716 (2018).

20. Meltzer, E. \& Schwartz, E. Schistosomiasis: current epidemiology and management in travelers. Curr. Infect. Dis. Rep. 15, 211-215 (2013).

21. Visser, L. G., Polderman, A. M. \& Stuiver, P. C. Outbreak of schistosomiasis among travelers returning from Mali, West Africa. Clin. Infect. Dis. 20, 280-285 (1995).

22. Sousa, M. S. et al. Performance of an ultra-sensitive assay targeting the circulating anodic antigen (CAA) for detection of Schistosoma mansoni infection in a low endemic area in Brazil. Front. Immunol. 10, 682 (2019).

23. Kariuki, T. M. et al. Parameters of the attenuated schistosome vaccine evaluated in the olive baboon. Infect. Immun. 72, 5526-5529 (2004).

24. Corstjens, P. L. et al. Tools for diagnosis, monitoring and screening of Schistosoma infections utilizing lateral-flow based assays and upconverting phosphor labels. Parasitology 141, 1841-1855 (2014).

25. Colley, D. G. et al. A five-country evaluation of a point-of-care circulating cathodic antigen urine assay for the prevalence of Schistosoma mansoni. Am. J. Trop. Med. Hyg. 88, 426-432 (2013).

26. Caldas, I. R. et al. Human Schistosoma mansoni: immune responses during acute and chronic phases of the infection. Acta Trop. 108, 109-117 (2008).

27. Thompson, L. J. et al. Conditioning of naive $\mathrm{CD} 4\left(^{+}\right) \mathrm{T}$ cells for enhanced peripheral Foxp3 induction by nonspecific bystander inflammation. Nat. Immunol. 17, 297-303 (2016).

28. Kariuki, T. M. \& Farah, I. O. Resistance to re-infection after exposure to normal and attenuated schistosome parasites in the baboon model. Parasite Immunol. 27, 281-288 (2005).

29. Soonawala, D., Geerts, J. W., de Mos, M., Yazdanbakhsh, M. \& Visser, L. G. The immune response to schistosome antigens in formerly infected travelers. Am. J. Trop. Med. Hyg. 84, 43-47 (2011).

30. Parrino, T. A., Schreiber, D. S., Trier, J. S., Kapikian, A. Z. \& Blacklow, N. R. Clinical immunity in acute gastroenteritis caused by Norwalk agent. N. Engl. J. Med. 297, 86-89 (1977).

31. Sombetzki, M. et al. Host defense versus immunosuppression: unisexual infection with male or female Schistosoma mansoni differentially impacts the immune response against invading cercariae. Front. Immunol. 9, 861 (2018).

32. Richter, D., Harn, D. A. \& Matuschka, F. R. The irradiated cercariae vaccine model: looking on the bright side of radiation. Parasitol. Today 11, 288-293 (1995)

33. Agnew, A. et al. The relationship between worm burden and levels of a circulating antigen (CAA) of five species of Schistosoma in mice. Parasitology 111, 67-76 (1995)

Publisher's note Springer Nature remains neutral with regard to jurisdictional claims in published maps and institutional affiliations.

๑ The Author(s), under exclusive licence to Springer Nature America, Inc. 2020 


\section{Methods}

Study design. This phase I trial (ClinicalTrials.gov identifier: NCT02755324) was an open-label dose-escalation study at the Leiden University Medical Center (LUMC).

Male cercariae were produced as previously reported ${ }^{34}$. The initial dose was 10 cercariae $(n=3)$, which was escalated to $30(n=3)$ and subsequently de-escalated to 20 cercariae $(n=3)$ on the basis of AEs. The 20 cercariae infection was then validated in another group $(n=8)$. The viability of cercariae was confirmed by imaging of cercariae penetrating skin explant $\mathrm{s}^{35}$. The cercariae were applied to the volunteer's forearm in $0.5-1 \mathrm{ml}$ of water for $30 \mathrm{~min}$, the number of remaining cercariae were counted by microscopy. Volunteers were observed for $30 \mathrm{~min}$ after exposure.

Volunteers were followed bi-weekly between week 0-24 and on week 52 . During each visit, AEs were recorded. Symptoms of an acute schistosomiasis syndrome were defined as moderate to severe symptoms of malaise, fatigue, fever, night sweats, flu-like symptoms or headache, between 2-7 weeks after exposure. Safety reports were reviewed regularly by an external safety monitor, who advised on dose escalation. Blood and urine samples were collected at all visits.

The pre-patent period was defined as the time until serum CAA levels were above $1.0 \mathrm{pg} \mathrm{ml}^{-1}$ (ref. ${ }^{24}$ ). At week 12 all volunteers were treated with $40 \mathrm{mg} \mathrm{kg}^{-1}$ praziquantel in two doses. A second regimen of $60 \mathrm{mg} \mathrm{kg}^{-1}$ praziquantel in two doses was provided if serum CAA levels persisted 3-6 weeks after treatment. Cure was defined as serum CAA levels $\leq 0.5 \mathrm{pg} \mathrm{ml}^{-1}$.

The study was approved by the LUMC Institutional Medical Ethical Research Committee (Institutional Review Board P16.111). It was performed according to the European Clinical Trial Directive 2001/20/EC, in accordance with ICH-GCP guidelines and the Declaration of Helsinki ${ }^{36,37}$.

Informed consent procedure. Healthy 18-45-year-old Schistosoma-naive volunteers were screened by medical history, general physical examination and safety laboratory tests. Informed consent was obtained from all volunteers.

Through advertisements, volunteers provided their email address and received written information. When they so wished, volunteers could schedule a screening visit at least $3 \mathrm{~d}$ after having received the information. They were then requested to complete an application form, which included a questionnaire regarding their health.

During the 1.5-2-h screening visit, the study purpose and procedures were explained and questions answered. The possible AEs and right of withdrawal was explained to the volunteers. The informed consent form was signed and a full physical exam was performed. All volunteers were required to consent to an HIV, hepatitis B (HBV) and hepatitis C (HCV) serological screening, urine toxicology and (for females), a pregnancy test at screening.

At the infection day ( $14 \mathrm{~d}$ to 23 weeks after screening), informed consent was reconfirmed, and a final check of inclusion and exclusion criteria was performed, including a focused physical exam. Volunteers were exposed to male Schistosoma mansoni cercariae after baseline assessment and safety laboratory tests.

The first volunteer was included on 27 October 2015 and the last volunteer was included on 1 February 2018

All three volunteers gave permission to use the photographs (Fig. 2a-c) taken of their skin after cercarial exposure for publication.

Inclusion and exclusion criteria. Inclusion criteria.

- Volunteer is aged $\geq 18$ and $\leq 45$ years and is in good health.

- Volunteer has adequate understanding of the procedures of the study and agrees to abide strictly thereby.

- Volunteer is able to communicate well with the investigator and is available to attend all study visits.

- Volunteer will remain within Europe (excluding Corsica) during the study period and is reachable by mobile telephone from week 3 to week 12 of the study period.

- Volunteer agrees to refrain from blood donation to Sanquin or for other purposes throughout the study period.

- For females: volunteer agrees to use adequate contraception and not to breastfeed for the duration of study.

- Volunteer has signed informed consent form.

Exclusion criteria.

- Any history, or evidence at screening, of clinically significant symptoms, physical signs or abnormal laboratory values suggestive of systemic conditions, such as cardiovascular, pulmonary, renal, hepatic, neurological, dermatological, endocrine, malignant, hematological, infectious, immune-deficient, psychiatric and other disorders, which could compromise the health of the volunteer during the study or interfere with the interpretation of the study results. These include, but are not limited to, any of the following:

- body weight $<50 \mathrm{~kg}$ or body mass index $<18$ or $>30 \mathrm{~kg} \mathrm{~m}^{-2}$ at screening;

- positive HIV, HBV or HCV screening tests:

- the use of immune-modifying drugs within 3 months before study onset (inhaled and topical corticosteroids and oral anti-histamines exempted) or expected use of such during the study period;
- history of malignancy of any organ system (other than localized basal cell carcinoma of the skin), treated or untreated, within the past 5 years;

- any history of treatment for severe psychiatric disease by a psychiatrist in the past year;

- history of drug or alcohol abuse interfering with normal social function in the period of 1 year before study onset;

- any clinically significant abnormalities (including extended QT interval) on electrocardiogram.

- The chronic use of any drug known to interact with praziquantel, artesunate or lumefantrine (artesunate combined with lumefantrine served as alternative treatment of schistosomiasis in an earlier phase of infection) metabolism (for example, phenytoin, carbamazepine, phenobarbital, primidone, dexamethasone, rifampicin, cimetidine, flecainide, metoprolol, imipramine, amitriptyline, clomipramine, class IA and III anti-arrythmics, antipsychotics, antidepressants, macrolides, fluorquinolones, imidazole- and triazole antimycotics and anti-histamines). Because lumefantrine may cause extension of QT-time, chronic use of drugs with effect on QT interval are excluded from the study.

- For female volunteers: positive urine pregnancy test at screening

- Any history of schistosomiasis or treatment for schistosomiasis

- Positive serology for schistosomiasis or elevated serum or urine CAA at baseline

- Known hypersensitivity to or contraindications (including co-medication) for use of praziquantel, artesunate or lumefantrine

- Being an employee or student of the Department of Parasitology or Infectious Diseases of the LUMC

\section{Adverse events. Grading.}

- Mild (grade 1): awareness of symptoms that are easily tolerated and do not interfere with usual daily activity

- Moderate (grade 2): discomfort that interferes with or limits usual daily activity

- Severe (grade 3): disabling, with subsequent inability to perform usual daily activity, resulting in absence or required bed rest

- Serious AE: any untoward medical occurrence in a patient or trial participant, which does not have a causal relationship with the treatment, and:

- is fatal, and/or

- is life-threatening for the volunteer, and/or

- makes hospital admission or an extension of the admission necessary, and/or

- causes persistent or significant invalidity or work disability, and/or

- manifests itself in a congenital abnormality or malformation, and/or

- could, according to the person that carries out the research, have developed to a serious undesired medical event, but was, however, prevented due to premature interference.

\section{Causality. Unrelated.}

- Not related: a relationship to the administration of the S. mansoni male cercariae cannot be reasonably established; another etiology is known to have caused the AE or is highly likely to have caused it.

- Unlikely related: a relationship to the administration of S. mansoni male cercariae is unlikely; however, it cannot be ruled out.

Related.

- Possibly related: there is a potential association between the event and administration of the S. mansoni male cercariae; however, there is an alternative etiology that is more likely.

- Probably related: administration of the $S$. mansoni male cercariae is the most likely cause; however, there are alternative reasonable explanations, even though less likely.

- Definitely related: administration of the S. mansoni male cercariae is the cause; another etiology causing the adverse event is not known.

Parasitological assays. CAA was measured in serum and urine by the previously described upconverting phosphor lateral flow (UCP-LF CAA) assay ${ }^{24,38}$. The presence of urine CCA was determined by the POC-CCA (Rapid Medical Diagnostics). The Schistosoma PCR on feces was performed according to previous descriptions (ISO 15189:2012-certified) ${ }^{39}$.

Antigen detection assays. Upconverting phosphor lateral flow assay for circulating anodic antigen. Both serum and urine were analyzed for schistosome CAA using a UCP-LF CAA assay. The assay was performed as described previously $y^{24,38}$. In brief, $500 \mu \mathrm{l}$ of serum (or $4 \mathrm{ml}$ of urine) samples or standards were diluted 1:1 in $4 \%$ trichloro-acetic acid (TCA) (or diluted 5:1 in 12\% TCA for urine samples) and incubated for $5 \mathrm{~min}$ at room temperature. Serum samples were centrifuged for $10 \mathrm{~min}$ at 13,000 r.p.m. (and urine samples were centrifuged for $45 \mathrm{~min}$ at 
4,000 r.p.m.). Then $500 \mu \mathrm{l}$ of supernatants of serum (or $4 \mathrm{ml}$ of urine) was applied to $0.5-\mathrm{ml}$ (or 4.0-ml) Amicon filtration devices (Amicon Ultra-0.5 (or Amicon Ultra4), Millipore) and concentrated to approximately $20 \mu \mathrm{l}$ by centrifugation for $25 \mathrm{~min}$ at 13,000 r.p.m. for serum (or $60 \mathrm{~min}$ at 4,000 r.p.m. for urine). The concentrates were diluted 1:5 in LF assay buffer and incubated in microtiter plate wells at $37^{\circ} \mathrm{C}$ for $1 \mathrm{~h}$ while shaking. LF strips were inserted into the wells and incubated for $3 \mathrm{~h}$ or overnight, before being read on a modified Packard FluoroCount microtiter plate reader ${ }^{24}$. A TCA-soluble fraction of $S$. mansoni adult worm antigen with known CAA concentration was used as a reference standard for the quantification of the antigen. Predefined cutoff values were used, where a serum CAA concentration above $1 \mathrm{pg} \mathrm{ml}^{-1}$ was defined as positive, below $0.5 \mathrm{pg} \mathrm{ml}^{-1}$ as negative and between 0.5 and 1.0 as undecisive $\mathrm{e}^{24}$.

Point-of-care circulating cathodic antigen. Urine samples were tested for the presence of schistosome CCA using a commercially available rapid diagnostic test (POC-CCA, batch no. 170622073, Rapid Medical Diagnostics) according to the manufacturer's procedure. Readings above a trace line were considered as positive.

Immune responses. Clinical diagnostics. Schistosome-specific antibodies, IgM against adult worms and anti-SEA IgG were determined according to ISO 15189:2012-certified routine diagnostic in-house IFA and ELISA respectively, which have been in use for the clinical diagnosis of schistosomiasis at the LUMC for decades ${ }^{40,41}$

IgM adult worm antibodies by IFA. IgM antibodies against adult worms were detected by an in-house IFA assay as previously described ${ }^{42}$. This IFA, as well as the ELISA described below, are currently in use at LUMC as the routine antibody detecting assays for the diagnosis of imported schistosomiasis and feature in all laboratory quality assessment requirements (ISO 15189:2012-certified), including successful participation in an external quality assessment scheme (UK-NEQAS). In brief, sections of Rossman's fixed male adult worms were incubated with a twofold dilution series of serum samples starting at 1:8 dilution. Following incubation with goat anti-human IgM (u-chain specific)-FITC antibody (Sigma-Aldrich; F5384), slides were examined using a fluorescence microscope. A negative control and a positive reference serum were run in parallel at each slide. The titer was determined as the dilution of the sample at which the fluorescence of adult worm gut epithelium was still visible. Samples were considered positive if titers were above 1:8. Previous studies showed that the IgM detected in this IFA is mainly directed against $\mathrm{CCA}^{42}$.

IgG against soluble egg antigen. IgG antibodies directed against $S$. mansoni SEA were detected by a previously described in-house ELISA with some minor modifications ${ }^{40}$. Crude SEA was prepared from S. mansoni eggs collected from the livers of infected hamsters ${ }^{43,44}$. A concentration of $5 \mu \mathrm{g}$ protein $\mathrm{ml}^{-1}$ was diluted in $100 \mu \mathrm{l}$ of $0.1 \mathrm{M}$ sodium carbonate buffer ( $\mathrm{pH} 9.6$ ) and coated overnight at $4{ }^{\circ} \mathrm{C}$ in 96 -well plates (Polysorb NUNC). Plates were stored at $-80^{\circ} \mathrm{C}$ until use. After thawing, plates were washed and blocked in 1\% BSA in PBS for $1 \mathrm{~h}$ at $37^{\circ} \mathrm{C}$. A dilution series of serum samples from $1: 16$ to $1: 2,048$ in a solution with $5 \%$ FCS and $0.05 \%$ Tween in PBS were incubated for $1 \mathrm{~h}$ at $37^{\circ} \mathrm{C}$. Plates were washed and incubated for $1 \mathrm{~h}$ at $37^{\circ} \mathrm{C}$ with mouse anti-human IgG alkaline phosphatase 1:10,000 (Sigma-Aldrich; A2064) in 4\% BSA and 0.05\% Tween/PBS Para-nitrophenyl phosphate (pNPP) substrate (Sigma-Aldrich; P5994) in $0.1 \mathrm{M}$ diethanolamine buffer ( $\mathrm{pH}$ 9.6) (Merck) was added for $1 \mathrm{~h}$ at room temperature after washing. Plates were read with the Multiskan EX reader at $405 \mathrm{~nm}$. A negative control and a positive reference serum were run in parallel at each plate. The titer was determined as the dilution of the sample at which the extinction is higher or equal to the reference standard. Samples were considered positive if titers were above 1:16.

Experimental immunological assays. Serum was analyzed for total IgE, S. mansoni AWA-specific IgE and IgG by ELISA ${ }^{45-47}$. Data were expressed as arbitrary units $\left(\mathrm{AU} \mathrm{ml} \mathrm{m}^{-1}\right.$ ). Peripheral blood mononuclear cells (PBMCs) were evaluated for their phenotype and function after 24-h stimulation with crude $S$. mansoni AWA by flow cytometry.

Adult worm antigen-specific IgE, IgG, IgG1 and IgG4. S. mansoni AWA-specific IgE and IgG was measured by ELISA modified from previous protocols ${ }^{45,46}$. In brief, adult $S$. mansoni worms were collected from hamsters and crude AWA was prepared as described previously ${ }^{48}$. MaxiSorp plates (Nunc) were coated overnight with $5 \mu \mathrm{g} \mathrm{ml}^{-1}$ AWA diluted in carbonate buffer $\mathrm{pH}$ 9.6. After blocking with $5 \%$ BSA/PBS, the sera were diluted with a solution with 5\% FCS and $0.05 \%$ Tween in PBS and the presence of IgG1 or IgG4 was shown by using horseradish peroxidase (HRP)-labeled anti-human IgG1 or HRP-labeled anti-human IgG4 (1:3,000 dilution; Sanquin). For measuring total anti-AWA IgG antibody, alkaline phosphatase-conjugated anti-human IgG (1:5,000 dilution; Sigma) was added, whereas for the anti-AWA IgE assay, the plate was incubated with biotinylated goat anti-human IgE (1:1,000 dilution; Vector Laboratories) followed by streptavidin HRP conjugate (1:10,000 dilution; Sanquin). IgG1 and IgG4 assays were developed using tetramethylbenzine, stopped with $10 \% \mathrm{H}_{2} \mathrm{SO}_{4}$ and the absorbencies were measured at $450 \mathrm{~nm}$. For total IgG and IgE, the color was developed by addition of pNPP (Sigma) diluted in diethanolamine buffer and optical density was measured at $405 \mathrm{~nm}$. For all four assays, the levels of antibody present in a given sample were expressed in $\mathrm{AU} \mathrm{ml}^{-1}$ or International Units ( $\mathrm{IU} \mathrm{ml}^{-1}$ ) according to the standard curve of pooled sera from inhabitants of an S. mansoni endemic area in Ghana. The levels of antibody present in a given samples were expressed in $\mathrm{AU} \mathrm{ml} \mathrm{m}^{-1}$ for total IgG, IgG1 and IgG4 or in IU ml ${ }^{-1}$ for IgE. Seroconversion was defined as antibody levels above $2 \times$ s.d. of the baseline.

Total IgE. Total IgE levels were measured as previously described ${ }^{47}$. Briefly, MaxiSorp plates were coated overnight with rabbit anti-human IgE (Dako). Plates were blocked with PBS 5\% BSA followed by incubation of diluted samples in PBS $0.05 \%$ Tween-20. As a reference, the World Health Organization standard of human serum IgE (NIBSC) was used, starting at a concentration of $90 \mathrm{IU} \mathrm{ml}^{-1}$. After a washing step, the plates were incubated with IgE biotinylated goat anti-human IgE antibody (Vector Laboratories) followed by an incubation with streptavidin alkaline phosphatase conjugate (Boehringer Mannheim). The color was developed by addition of pNPP (Boehringer Mannheim) diluted in diethanolamine buffer and optical density was measured at $405 \mathrm{~nm}$. The results were expressed in $\mathrm{IU} \mathrm{ml}^{-1}$. Seroconversion was defined as antibody levels above $2 \times$ s.d. of the baseline.

Ex vivo cytokines. Serum samples were tested for the presence of different cytokines using a commercially ProcartaPlex Multiplex Immunoassay (17-plex, lot 178863000 , Invitrogen) according to the manufacturer instructions. The following cytokines were measured: IFN- $\alpha$, IFN- $\beta$, IL-1 $\beta$, IL-10, IL-12p70, IL-13, IL-15, IL-2 IL-22, IL-23, IL-4, IL-6, IP-10, MCP-1, MIP- $1 \alpha$, MIP- $1 \beta$ and TNF- $\alpha$ using the Bioplex 200 Luminex (Bio-Rad).

Cellular immunology. Between week 0 and 24 after exposure every 4 weeks, human PBMCs were isolated from whole blood collected in heparin. Cells of two heparin tubes were diluted at least 1:2 with HBSS (ThermoFisher) at room temperature. Ten milliliters of ficoll at room temperature was added, followed by 25 min centrifugation at $400 \mathrm{~g}$ with low brake. Cells were collected and washed with HBSS, counted and frozen in 10\% DMSO, in RPMI Hepes (Invitrogen), with $100 \mathrm{U} \mathrm{ml}^{-1}$ penicillin, $100 \mu \mathrm{g} \mathrm{ml}^{-1}$ streptomycin, $1 \mathrm{mM}$ pyruvate $/ 2 \mathrm{mM}$ glutamate and $10 \%$ FCS (Bodinco). Subsequently, PBMCs were thawed in RPMI Hepes, with $100 \mathrm{U} \mathrm{ml}^{-1}$ penicillin, $100 \mu \mathrm{g} \mathrm{ml}^{-1}$ streptomycin, $1 \mathrm{mM}$ pyruvate $/ 2 \mathrm{mM}$ glutamate and $10 \% \mathrm{FCS}$ and rested overnight at $37^{\circ} \mathrm{C}$ with $5 \% \mathrm{CO}_{2}$. Cells were counted and transferred to a 96-well round bottom plate (Corning) with 500,000 cells per well. Cells were stimulated with AWA $\left(50 \mu \mathrm{g} \mathrm{ml}^{-1}\right)$ for $24 \mathrm{~h}$. Staphylococcal enterotoxin B (SEB) (Sigma-Aldrich) $200 \mathrm{ng} \mathrm{ml}^{-1}$ was used as a positive control and RPMI Hepes (Invitrogen), with $100 \mathrm{U} \mathrm{ml}^{-1}$ penicillin, $100 \mu \mathrm{g} \mathrm{ml}^{-1}$ streptomycin, $1 \mathrm{mM}$ pyruvate $/ 2 \mathrm{mM}$ glutamate and $10 \% \mathrm{FCS}$ as a negative control. After $4 \mathrm{~h}$ of incubation $5 \mathrm{mg} \mathrm{ml}^{-1}$ brefeldin A (Sigma) was added to SEB-stimulated wells and after $20 \mathrm{~h}$ to AWA- and medium-stimulated wells. After a total stimulation of $24 \mathrm{~h}$, the cells were stained with Aqua (Invitrogen) and fixed with $3.9 \%$ formaldehyde (Sigma). After fixating, the cells were stained with the following antibodies: CD3, CD4, IFN- $\gamma$, IL-2, Th2-cytokines (IL-4, IL-5, IL-13), TNF and IL-10 (Supplementary Table 4). Human FC block was used to avoid nonspecific interactions. The cells were measured with the FACSCanto II (BD Biosciences; Supplementary Fig. 1). The data were analyzed with FlowJo 10.5 software for MAC OS. The gating was placed with the help of fluorescence minus one controls, the medium as a negative control and SEB as a positive control. The leftover, aquastained and fixed cells were frozen in 10\% DMSO, in RPMI Hepes (Invitrogen), with $100 \mathrm{U} \mathrm{ml}^{-1}$ penicillin, $100 \mu \mathrm{g} \mathrm{ml}^{-1}$ streptomycin, $1 \mathrm{mM}$ pyruvate $/ 2 \mathrm{mM}$ glutamate and $10 \%$ FCS (Bodinco) and stored at $-80^{\circ} \mathrm{C}$. The cells were thawed at $37^{\circ} \mathrm{C}$ and stained with the following antibodies: CD3, CD4, CD25, CD127 and FOXP3 (Supplementary Table 4). As before, human FC block was used to avoid nonspecific interactions. The cells were measured with the FACSCanto II (BD Biosciences; Supplementary Fig. 1). The data were analyzed with FlowJo 10.5 software for MAC OS. The gating was placed with help of fluorescence minus one controls, the medium as a negative control and SEB as a positive control.

Statistical analysis. All 17 volunteers were included in the intention-to-treat analysis (safety analysis and parasitological assays). One volunteer was excluded from the per-protocol analysis (all immunological readouts) on the basis of high baseline AWA-specific IgG and IgG1 levels. Samples from individual volunteers were measured once and plotted as single values.

Demographics and the presence of symptoms between groups were analyzed with a Mann-Whitney $U$-test, time to patency with a log-rank test and correlations with a Spearman's rank test. Changes in the frequency of cytokine-producing cells over time were analyzed using a linear mixed model. Time was considered as the fixed effect and the volunteer ID as a random effect for the intercept. $P$ values based on Student's $t$-tests were obtained using the Satterthwaite's degrees of freedom method. In the cytokine boxplots, the negative values (after subtracting the medium condition) were set to zero to prevent a negative cytokine response. However, the statistical analysis was performed on the unaltered data. All statistical tests were two-tailed with $\alpha$ set at the 0.05 level. 
Analyses were performed using IBM SPSS statistics for Windows, v.23.0 (IBM Corp.) and R (v.3.5). The R packages used were ggplot2, lme4 and lmerTest ${ }^{49-51}$. $P$ values were considered significant when $P<0.05$.

Mixomics and data integration. Data integration was performed using the mixOmics package in $\mathrm{R}$ (v.6.8.0 $)^{52,53}$. This method allowed us to correlate across datasets, while associating features with outcome. Feature selection was performed using Lasso-like penalization for each of the datasets. The number of components was set at two and tuning was performed to find the minimum number of features needed per dataset (in a range from 1-3 per component) and the correlation between datasets was entered into the design matrix. The number of features included in the final model was 3, 3, 2 and 5 for cytokines, antigens, antibodies and cellular responses, respectively. A correlation of 0.75 between datasets was used in the design matrix.

Extended information can be found in the Nature Research Reporting Summary.

Reporting Summary. Further information on research design is available in the Nature Research Reporting Summary linked to this article.

\section{Data availability}

The data that support this publication are available at ImmPort (https://www. immport.org) under study accession SDY1609. All data will be made available for further research, provided that reference is made to the LUMC source.

\section{References}

34. Janse, J. J. et al. Establishing the production of male Schistosoma mansoni cercariae for a controlled human infection model. J. Infect. Dis. 218, $1142-1146$ (2018)

35. Winkel, B. M. F. et al. Early induction of human regulatory dermal antigen presenting cells by skin-penetrating Schistosoma mansoni cercariae. Front. Immunol. 9, 2510 (2018).

36. World Medical Association. World Medical Association Declaration of Helsinki: ethical principles for medical research involving human subjects. JAMA 310, 2191-2194 (2013).

37. European Commission. Clinical Trials - Directive 2001/20/EC. Official Journal of the European Communities https://ec.europa.eu/health/sites/health/ files/files/eudralex/vol-1/dir_2001_20/dir_2001_20_en.pdf (2001).

38. Corstjens, P. L. et al. Improved sensitivity of the urine CAA lateral-flow assay for diagnosing active Schistosoma infections by using larger sample volumes. Parasit. Vectors 8, 241 (2015).

39. Obeng, B. B. et al. Application of a circulating-cathodic-antigen (CCA) strip test and real-time PCR, in comparison with microscopy, for the detection of Schistosoma haematobium in urine samples from Ghana. Ann. Trop. Med. Parasitol. 102, 625-633 (2008).

40. Deelder, A. M. et al. Applicability of different antigen preparations in the enzyme-linked immunosorbent assay for Schistosomiasis mansoni. Am. J. Trop. Med. Hyg. 29, 401-410 (1980).

41. Nash, T. E., Ottesen, E. A. \& Cheever, A. W. Antibody response to a polysaccharide antigen present in the schistosome gut. II. Modulation of antibody response. Am. J. Trop. Med. Hyg. 27, 944-950 (1978).

42. Deelder, A. M., van Zeyl, R. J., Fillie, Y. E., Rotmans, J. P. \& Duchenne, W. Recognition of gut-associated antigens by immunoglobulin $\mathrm{M}$ in the indirect fluorescent antibody test for Schistosoma mansoni. Trans. R. Soc. Trop. Med. Hyg. 83, 364-367 (1989).

43. Dalton, J. P., Day, S. R., Drew, A. C. \& Brindley, P. J. A method for the isolation of schistosome eggs and miracidia free of contaminating host tissues. Parasitology 115, 29-32 (1997).

44. Deelder, A. M. Immunology of experimental infections with Schistosoma mansoni in the Swiss mouse and with Fasciola hepatica in the rabbit. Acta Leiden 39, 5-107 (1973).

45. van den Biggelaar, A. H., Borrmann, S., Kremsner, P. \& Yazdanbakhsh, M. Immune responses induced by repeated treatment do not result in protective immunity to Schistosoma haematobium: interleukin (IL)-5 and IL-10 responses. J. Infect. Dis. 186, 1474-1482 (2002).
46. Faulkner, H. et al. Antibody responses in onchocerciasis as a function of age and infection intensity. Parasite Immunol. 23, 509-516 (2001).

47. Staal, S. L. et al. Prevalence of atopy following mass drug administration with albendazole: a study in school children on Flores Island, Indonesia. Int. Arch. Allergy Immunol. 177, 192-198 (2018).

48. van Dam, G. J. et al. Antibody response patterns against Schistosoma mansoni in a recently exposed community in Senegal. J. Infect. Dis. 173, 1232-1241 (1996).

49. Bates, D., Machler, M., Bolker, B. M. \& Walker, S. C. Fitting linear mixedeffects models using lme4. J. Stat. Softw. 67, 1-48 (2015).

50. Kuznetsova, A., Brockhoff, P. B. \& Christensen, R. H. B. lmerTest package: tests in linear mixed effects models. J. Stat. Softw. 82, 1-26 (2017).

51. Wickham, H. ggplot2: Elegant Graphics for Data Analysis 2nd edn (Springer, 2016).

52. Rohart, F., Gautier, B., Singh, A. \& Le Cao, K. A. mixOmics: an R package for omics feature selection and multiple data integration. PLoS Comput. Biol. 13 e1005752 (2017)

53. Singh, A. et al. DIABLO: an integrative approach for identifying key molecular drivers from multi-omic assays. Bioinformatics 35, 3055-3062 (2019)

\section{Acknowledgements}

We thank P. van Genderen for reviewing the safety data and providing his advice as safety monitor of our study. We thank M. Casacuberta Partal, M.A.A. Erkens, J.L. FehrmannNaumann, M.S. Ganesh, H. Gerritsma, G.C. Hardeman, P.T. Hoekstra-Mevius, Y.C.M. Kruize, Y.D. Mouwenda, H.H. Smits, K. Suijk-Benschop, J.J.C. de Vries and C.J.G. van Zeijl-van der Ham for their laboratory, clinical and data-analyzing support during the study. Most of all we thank all volunteers participating in the study, without whom the study could not have been performed. Dr. Roestenberg was supported by a Veni grant (no. 016.156.076) from the Netherlands Organization for Health Research and Development and a Gisela Thier Fellowship (no. 14-0645) from LUMC. Dr. Langenberg and Dr. Hoogerwerf were supported by a grant from Dioraphte Foundation (no. 16020405). The funding sources had no role in collecting, analyzing, interpreting or reporting the data. Dr. Jochems has received funding from the European Union's Horizon 2020 Research and Innovation Programme under Marie Skłodowska-Curie grant agreement no. 707404. The opinions expressed in this document reflect only the author's view. The European Commission is not responsible for any use that may be made of the information that it contains.

\section{Author contributions}

M.C.C.L., M.-A.H., J.P.R.K., L.G.V. and M.R. were the clinical investigators. J.J.J. performed the trial data management. P.H.V.-M. was the trial nurse and responsible for clinical quality assurance. J.J.J., J.K.-v.O., C.F., A.O.-F., M.T.v.d.B., F.W.B.v.L., P.M., A.v.D. and C.H.H. were responsible for the production, quality assurance and quality control of infectious cercariae. C.J.d.D., R.v.S., M.D.M., E.S., B.M.F.W., L.v.L., G.J.v.D. and P.L.A.M.C. were responsible for parasitological testing, antibody and cellular assays M.C.C.L., G.J.v.D., C.H.H., M.Y., L.G.V. and M.R. prepared the research protocol and were involved in the study design. M.C.C.L., S.P.J., K.A.S. and M.R. performed the data analysis and statistical analysis. M.C.C.L. and M.R. wrote the manuscript. All authors reviewed the manuscript.

\section{Competing interests}

The authors declare no competing interests.

\section{Additional information}

Extended data is available for this paper at https://doi.org/10.1038/s41591-020-0759-x. Supplementary information is available for this paper at https://doi.org/10.1038/ s41591-020-0759-x.

Correspondence and requests for materials should be addressed to M.R.

Peer review information Alison Farrell was the primary editor on this article and managed its editorial process and peer review in collaboration with the rest of the editorial team.

Reprints and permissions information is available at www.nature.com/reprints. 
a

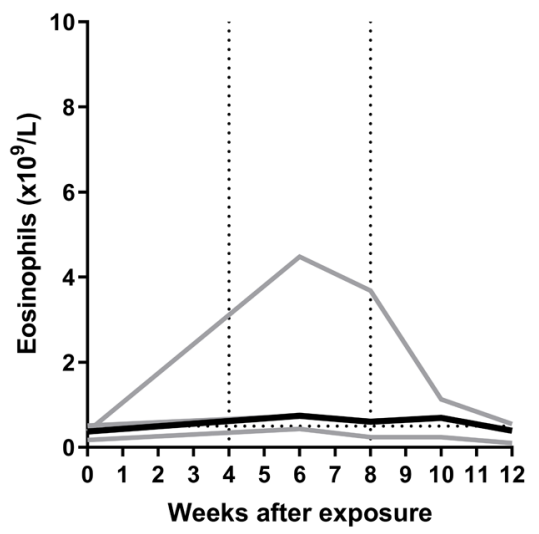

b

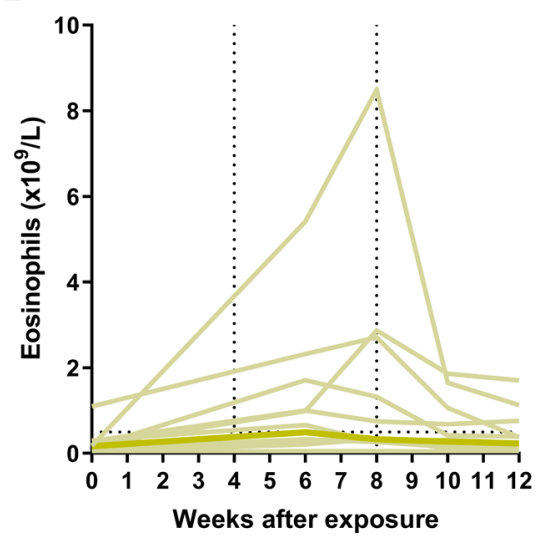

c

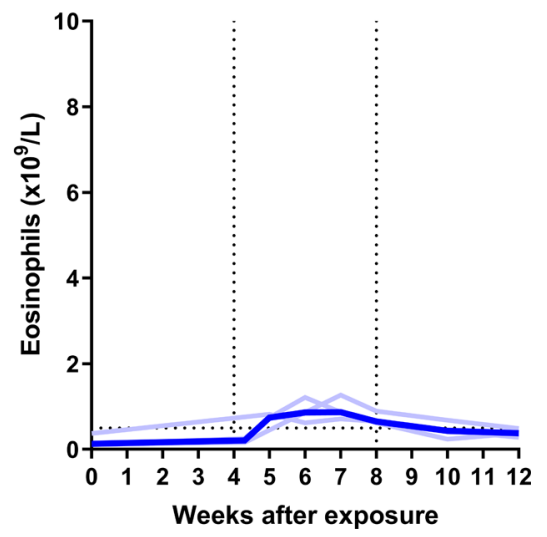

Extended Data Fig. 1 | Eosinophil counts. a-c. Eosinophil counts $\left(x 10^{\%} / \mathrm{L}\right)$ per volunteer and the median per group. The thin green, red, and blue lines represent data of individual volunteers infected with $10(n=3), 20(n=11)$ or $30(n=3)$ cercariae respectively, while thick lines represent the median of each group. 


\section{NATURE MEDICINE}
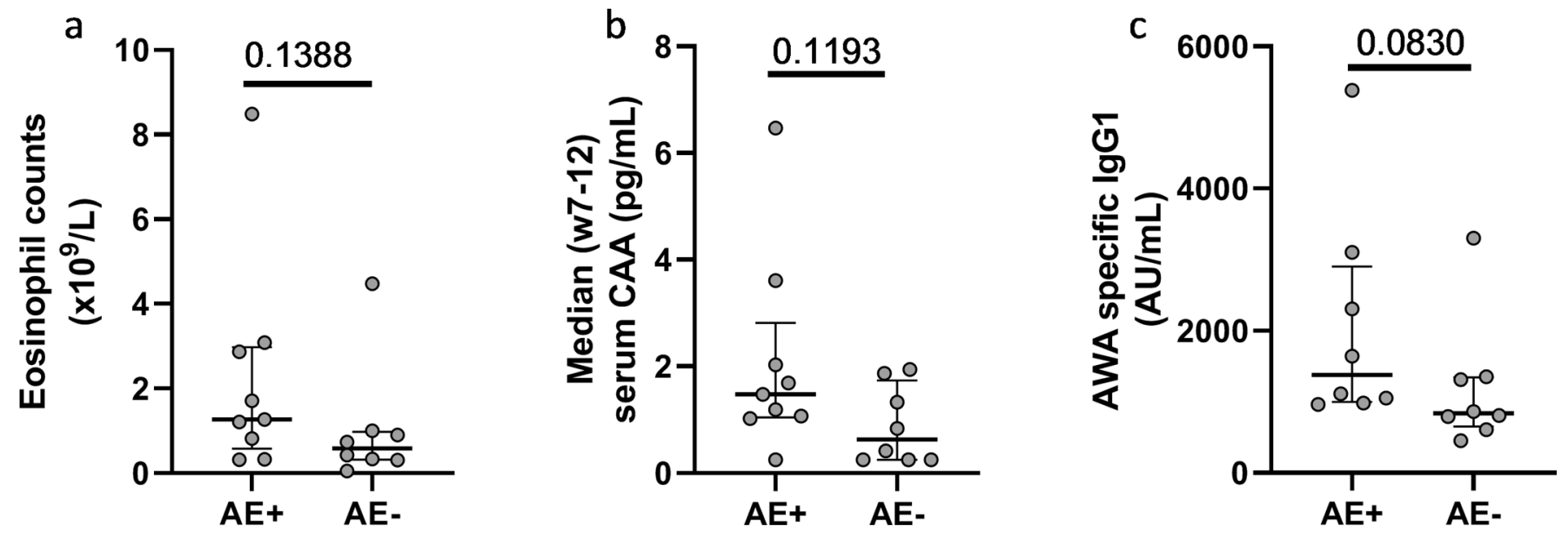

Extended Data Fig. 2 | Relation between symptoms of an acute schistosomiasis syndrome and immunological readouts. The relation between the presence of symptoms of an acute schistosomiasis infection and $\mathbf{a}$. the highest eosinophil count $(n=17), \mathbf{b}$. the median serum CAA level from week 7 to 12 $(n=17)$, or $c$. the AWA specific lgG1 response at week $16(n=16)$. All using the two-sided Mann-Whitney $U$ test. Individual data is presented as dots, the line represents the median, while the error bars represent the interquartile range of the groups. 
a

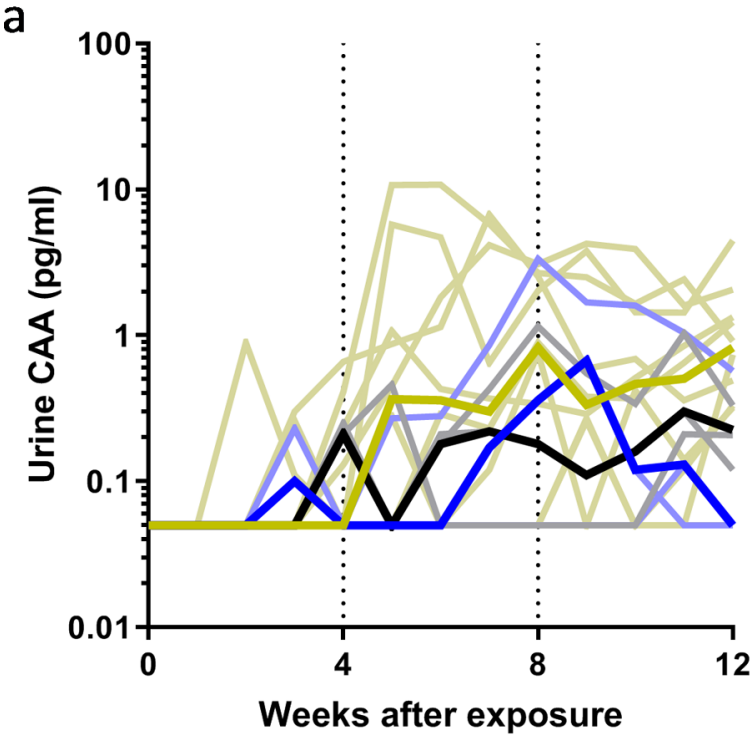

b

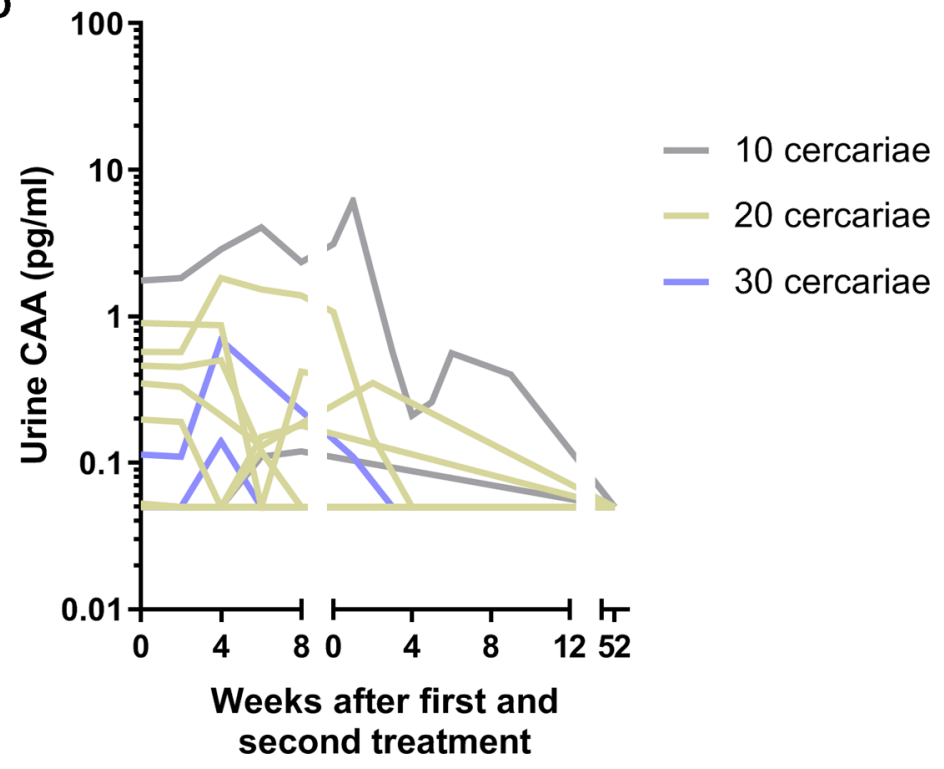

Extended Data Fig. 3 | Urine CAA levels. a. Urine CAA levels after exposure and $\mathbf{b}$. after first and second praziquantel treatment (week 0 ) and at week 52 after exposure. The thin green, red, and blue lines represent of individual volunteers infected with $10(n=3), 20(n=11)$ or $30(n=3)$ cercariae respectively, while thick lines represent the median of each group before treatment. 
a

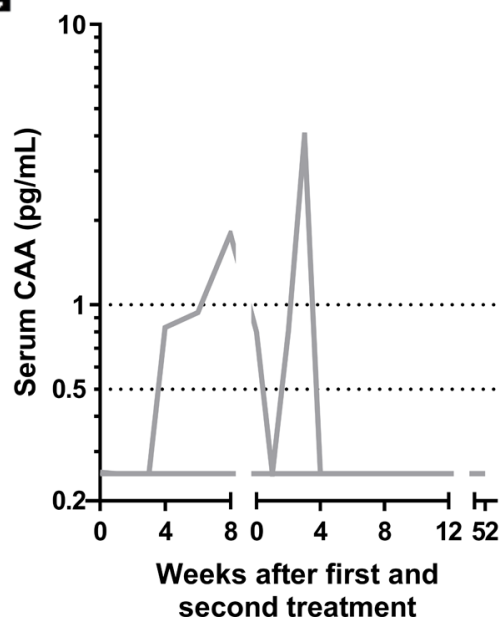

b

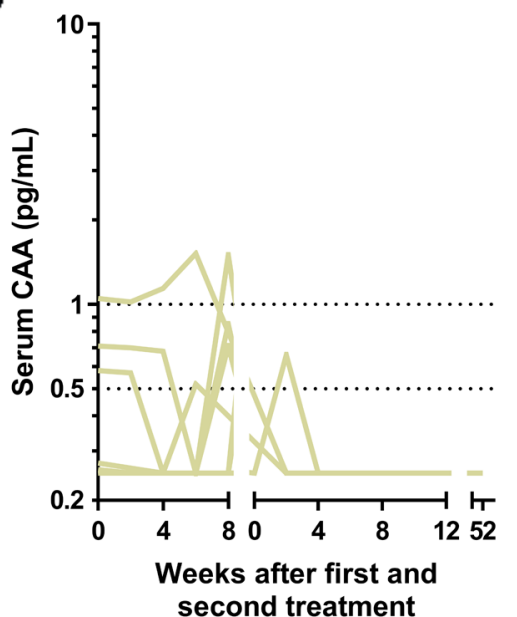

C

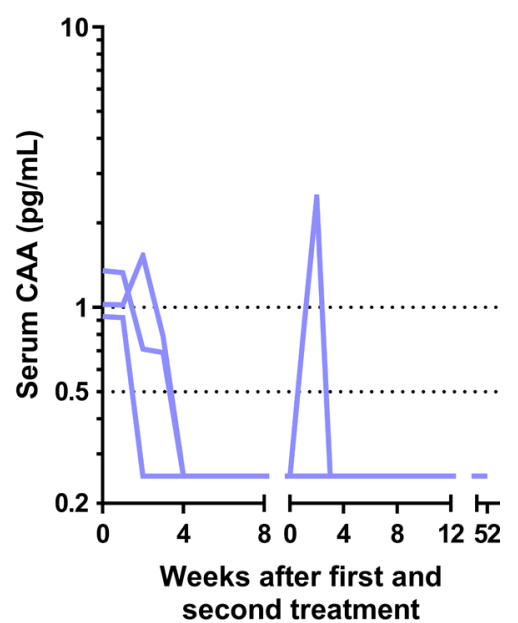

Extended Data Fig. 4 | Serum CAA levels after treatment. a-c. Serum CAA levels in pg/ml after the first treatment, second treatment, and at week 52. All values below the detection threshold of $0.5 \mathrm{pg} / \mathrm{mL}$, are plotted at $0.25 \mathrm{pg} / \mathrm{mL}$. The gray, yellow and blue lines represent data of individual volunteers infected with $10(n=3), 20(n=11)$ or $30(n=3)$ cercariae respectively. 
a

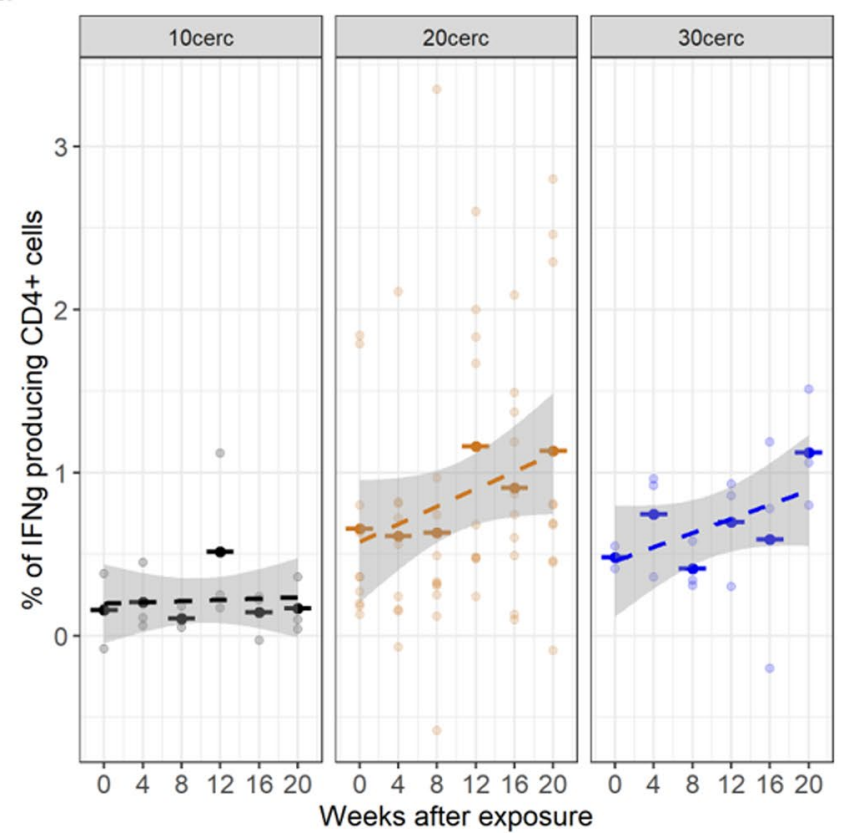

b

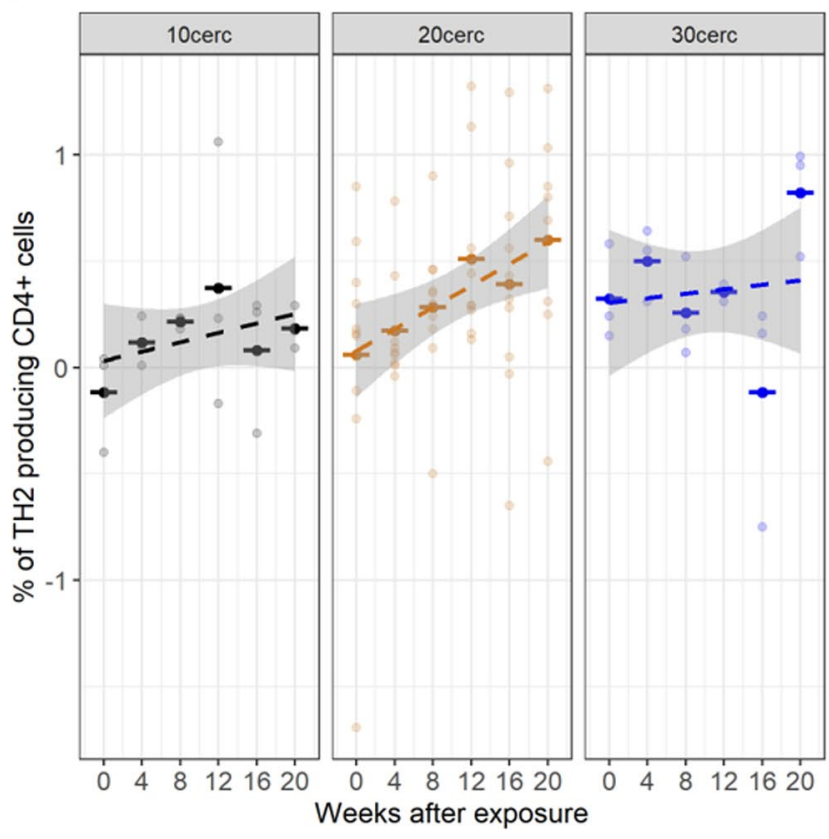

Extended Data Fig. 5 | IFNg and TH2 cytokine producing CD4 + T-cells over time. The percentage of $\mathbf{a}$. IFN $\gamma$ and $\mathbf{b}$. Th2-cytokine producing CD4+ T-cells over time in weeks after exposure in the 10 cercariae (gray, $n=3$ ), 20 cercariae (yellow, $n=10$ ) and 30 cercariae (blue, $n=3$ ) groups. Dotted lines are linear regression lines, gray areas are confidence intervals, light dots are individual data, and horizontal lines with dots are the average values. 
a

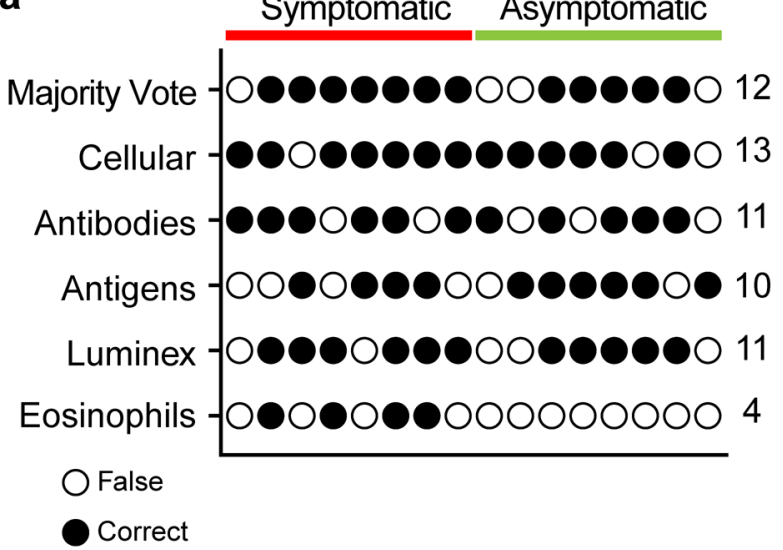

C

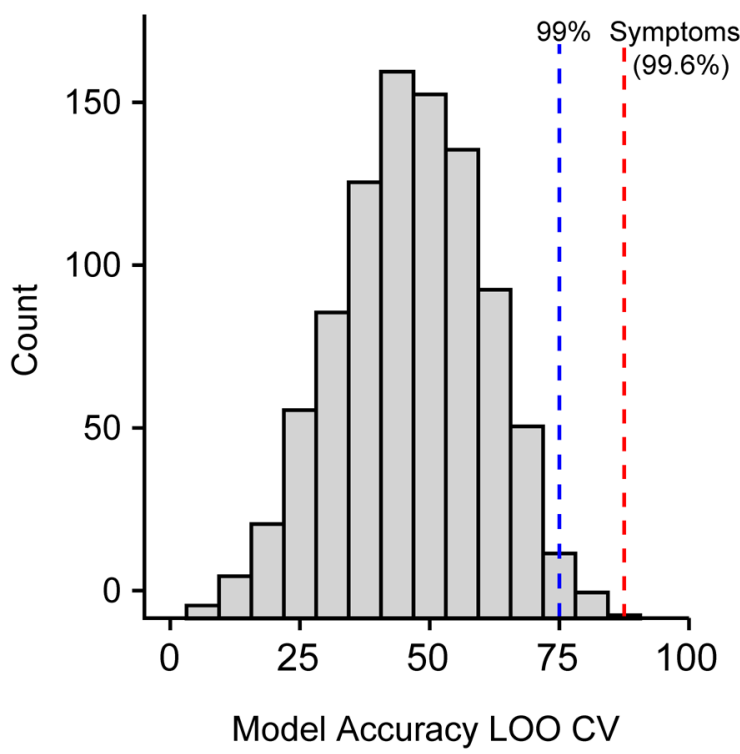

b

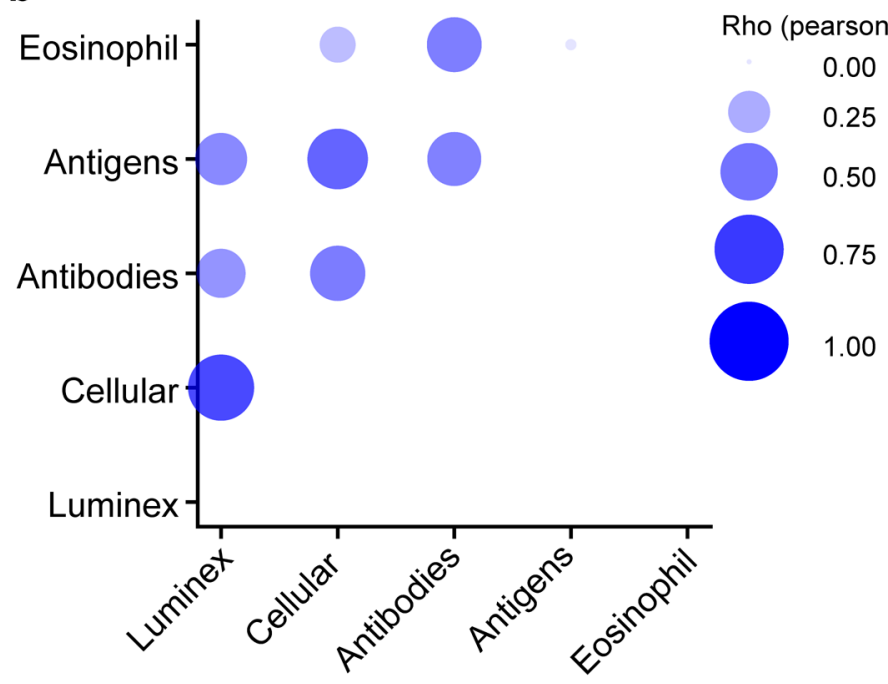

d

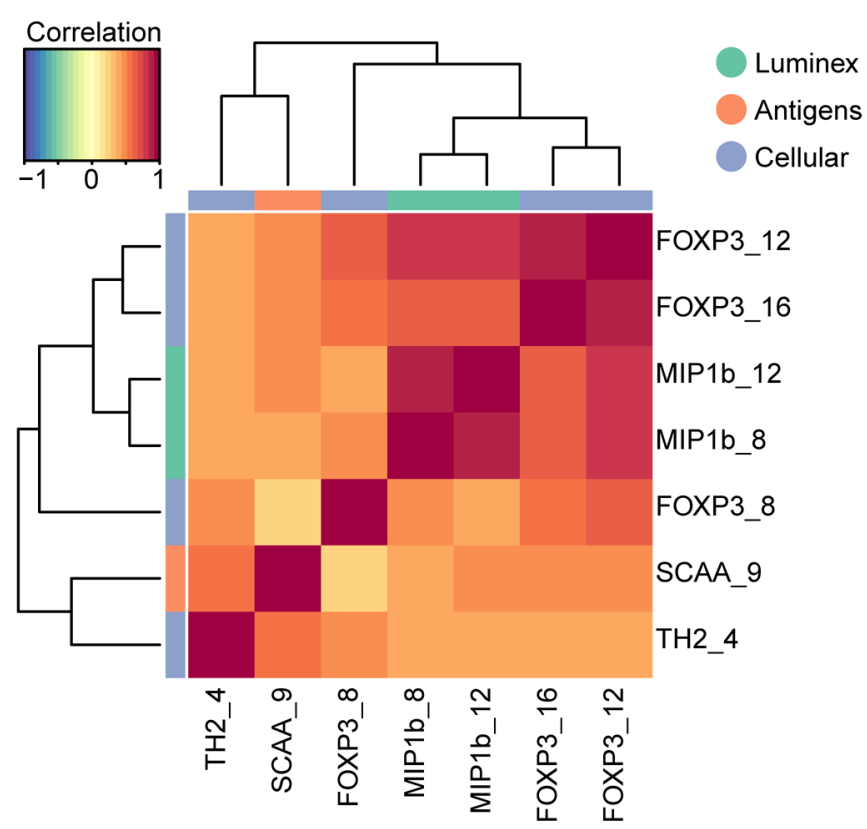

Extended Data Fig. 6 | Effect of eosinophil data and model performance. a. Individual predictions across folds for the full model per dataset including majority vote. Each symbol represents one prediction, with volunteers in columns and datasets in rows. Filled circles indicate a correct prediction and open circles a false prediction. Symptomatic and asymptomatic volunteers are indicated in red and the number of correct predictions per dataset is indicated. b. Mean Pearson correlation score between datasets using the first component of the projection onto the latent space across all folds from the model including all datasets. Size and color of circles reflect the mean rho value. c. Permutations analysis $(n=1000)$ with leave-one-out cross-validation on the full model using all subjects and including the four datasets without eosinophils. Blue and red dashed lines indicate the $99^{\text {th }}$ percentile and the accuracy when comparing symptomatic and asymptomatic individuals (99.6\%), respectively. d. Spearman correlation matrix of the seven consensus features selected in $>75 \%$ of folds in the leave-one-out cross-validation. Features were clustered using hierarchical clustering with complete linkage on Euclidean distance. All graphs are based on $n=16$. 


\section{Reporting Summary}

Nature Research wishes to improve the reproducibility of the work that we publish. This form provides structure for consistency and transparency in reporting. For further information on Nature Research policies, see Authors \& Referees and the Editorial Policy Checklist.

\section{Statistics}

For all statistical analyses, confirm that the following items are present in the figure legend, table legend, main text, or Methods section.

$\mathrm{n} / \mathrm{a}$ Confirmed

\ The exact sample size $(n)$ for each experimental group/condition, given as a discrete number and unit of measurement

$\square$ A statement on whether measurements were taken from distinct samples or whether the same sample was measured repeatedly

$\varnothing$ The statistical test(s) used AND whether they are one- or two-sided

Only common tests should be described solely by name; describe more complex techniques in the Methods section.

$\square$ A description of all covariates tested

$\triangle \square$ A description of any assumptions or corrections, such as tests of normality and adjustment for multiple comparisons

A full description of the statistical parameters including central tendency (e.g. means) or other basic estimates (e.g. regression coefficient)

$\triangle$ AND variation (e.g. standard deviation) or associated estimates of uncertainty (e.g. confidence intervals)

For null hypothesis testing, the test statistic (e.g. $F, t, r$ ) with confidence intervals, effect sizes, degrees of freedom and $P$ value noted

Give $P$ values as exact values whenever suitable.

Х $\square$ For Bayesian analysis, information on the choice of priors and Markov chain Monte Carlo settings

Х $\square$ For hierarchical and complex designs, identification of the appropriate level for tests and full reporting of outcomes

$\square$ Estimates of effect sizes (e.g. Cohen's $d$, Pearson's $r$ ), indicating how they were calculated

Our web collection on statistics for biologists contains articles on many of the points above.

\section{Software and code}

Policy information about availability of computer code

Data collection Castor EDC, version 2018.4

Data analysis IBM SPSS statistics for Windows, version 23.0 (IBM Corp., Armonk, NY, USA). R, version 3.5, with the R-packages used "ggplot2", "Ime4", "ImerTest" and "MixOmics". Flow cytometric analysis was performed using FlowJo 10.5 software for MAC OS.

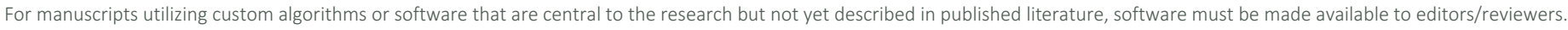
We strongly encourage code deposition in a community repository (e.g. GitHub). See the Nature Research guidelines for submitting code \& software for further information.

\section{Data}

Policy information about availability of data

All manuscripts must include a data availability statement. This statement should provide the following information, where applicable:

- Accession codes, unique identifiers, or web links for publicly available datasets

- A list of figures that have associated raw data

- A description of any restrictions on data availability

The datasets generated and analyzed during the current study will be made available through the immport data repository (https://www.immport.org). All data will be made available for further research, provided that reference is made to the LUMC source. 
Please select the one below that is the best fit for your research. If you are not sure, read the appropriate sections before making your selection.

$\bigotimes$ Life sciences $\quad \square$ Behavioural \& social sciences $\quad \square$ Ecological, evolutionary \& environmental sciences

For a reference copy of the document with all sections, see nature.com/documents/nr-reporting-summary-flat.pdf

\title{
Life sciences study design
}

All studies must disclose on these points even when the disclosure is negative.

Sample size For future vaccine proof-of-concept efficacy studies using the described human model for schistosomiasis, small groups of roughly 10 subjects are generally preferred, allowing for detection of roughly $50 \%$ protective vaccines with a power of $80 \%$ (alpha $<0.05,2$-tailed). A $100 \%$ infection rate in the infectivity control group is required to achieve this power. With a $90 \%$ infection rate $80 \%$ power is retained with an infection rate of $28 \%$ in the vaccine group. Therefore, we targeted a group size of 10 individuals and as high as possible infection rate. Small groups of 3 volunteers were used to pilot the safety and attack rate of infection. If the number of cercariae in a group was safe and $100 \%$ of volunteers was infected a second group, with 7 volunteers, was exposed to the same cercarial dose to reach the pre-calculated group of 10 volunteers as described above. Given the low number of individuals and the exclusion of one volunteer from all analysis (see data exclusions), the confirmatory cohort was amended to consist of 8 volunteers to maintain a fully evaluable group total of 10 individuals.

Data exclusions The data of one (out of 17) volunteer was excluded from the per-protocol analysis (all immunological read-outs) based on high adult worm antigen specific IgG and IgG1 levels before infection. The exclusion criteria of the per-protocol analysis was not pre-established. This volunteer was not excluded from the intention-to-treat analysis (safety analysis and parasitological assays).

Replication Each cercarial dose was applied to 3 or 11 volunteers in total. Blood and urine withdrawal were taken at pre-specified time points. In total 4 blood samples (out of 306 samples) and 5 urine samples (out of 306 samples) were missing. Experimental replication was not attempted

Randomization Individuals were not randomized, since the study followed an adaptive dose design. All groups were exposed sequentially, as such could not be randomized. given the small numbers co-variates could not be controlled.

Blinding

There was no blinding. All groups were exposed sequentially, as such could not be randomized. given the small numbers co-variates could not be controlled.

\section{Reporting for specific materials, systems and methods}

We require information from authors about some types of materials, experimental systems and methods used in many studies. Here, indicate whether each material, system or method listed is relevant to your study. If you are not sure if a list item applies to your research, read the appropriate section before selecting a response.

\begin{tabular}{l|l} 
Materials \& experimental sys \\
\hline n/a & Involved in the study \\
$\square$ & $\bigotimes$ Antibodies \\
$\square$ & $\square$ Eukaryotic cell lines \\
$\square$ Palaeontology \\
$\square$ Animals and other organisms \\
$\square$ Human research participants \\
$\square$ Clinical data
\end{tabular}

\begin{tabular}{l} 
Methods \\
\hline n/a Involved in the study \\
$\square$ \\
$\square$ ChIP-seq \\
$\square$ \\
$\square$ Frow cytometry
\end{tabular}

\section{Antibodies}

Antibodies used

\author{
Antibodies used in Flow cytometry: \\ IL-2 FITC BD Biosciences 340448, lot number 8136693 \\ IL-4 PE BD Biosciences 340451, , lot number 8239881 \\ IL-5 PE Biolegend 504303, lot number B261402 \\ II-13 PE Biolegend 501903, lot number B217729 \\ CD4 PerCP-ef710 eBioscience 46-0047, lot number 2023412 \\ TNF PE-Cy7 eBioscience 25-7349, lot number 4335153 \\ IL-10 APC BD Biosciences 554707, lot number 8092739 \\ CD3 APC ef780 eBioscience 47-0038, lot number 4331778 \\ IFNg BV421 Biolegend 502531, lot number B247533 \\ Human FC block - eBioscience 14-9161, lot number 4304475 \\ Live/Dead Aqua AmCyan hermoFisher L34957, lot number 2018290 \\ CD3 FITC BD Biosciences 555339, lot number 8333818 \\ CD4 Pe-Cy7 BD Biosciences 557852, lot number 8317967
}


CD25 BV421 BD Biosciences 562442, lot number 9095648

CD127 APCe780 eBioscience 47-1278-42, lot number 1978210

FOXP3 APC eBioscience 17-4776-42, lot number E509947-1653

Antibodies used in Luminex:

17-plex ProcartaPlex Multiplex Immunoassay Invitrogen, lot number 178863000

IFNa, IFNb, IL-1b, IL10, IL12p70, IL13, IL15, IL2, IL22, IL23, IL4, IL6, IP10, MCP1, MIP1a, MIP1b and TNFa

Antibodies used in Immuno fluorescence assay (IFA):

Goat anti-human IgM (u-chain specific)-FITC antibody (Sigma-Aldrich; F5384), Lot SLBH1186

Antibodies used in clinical diagnostic ELISA

Mouse anti human IgG alkaline phosphatase (Sigma-Aldric; A2064) Lot SLBR4951V

Antibodies used in experimental ELISA

Horseradish peroxidase (HRP-labelled anti human IgG1) (Sanquin, Amsterdam, the Netherlands), M1328, lot number 8000036002

HRP-labelled anti human IgG4 (Sanquin, Amsterdam, the Netherlands), M1331, lot number 8000037146

Alkaline phosphatase conjugated anti human IgG (Sigma, St Louis, MO, USA), A9544, lot number 10K4843

Biotinylated goat anti human IgE (Vector Laboratories, Burlingame, CA, USA), BA3040, lot number 10710

Streptavidin HRP conjugate (Sanquin, Amsterdam, the Netherlands),11.089.161.001, lot number 10865700

Rabbit anti-human IgE (Dako, Glostrup, Denmark), A00094, lot number 20035039

Validation

All antibodies were validated for use in flow cytometry (see manufacturer's website):

IL-2 FITC BD Biosciences 340448

IL-4 PE BD Biosciences 340451

IL-5 PE Biolegend 504303

II-13 PE Biolegend 501903

CD4 PerCP-ef710 eBioscience 46-0047

TNF PE-Cy7 eBioscience 25-7349

IL-10 APC BD Biosciences 554707

CD3 APC ef780 eBioscience 47-0038

IFNg BV421 Biolegend 502531

Human FC block - eBioscience 14-9161

Live/Dead Aqua AmCyan hermoFisher L34957

CD3 FITC BD Biosciences 555339, lot number 8333818

CD4 Pe-Cy7 BD Biosciences 557852, lot number 8317967

CD25 BV421 BD Biosciences 562442, lot number 9095648

CD127 APCe780 eBioscience 47-1278-42, lot number 1978210

FOXP3 APC eBioscience 17-4776-42, lot number E509947-1653

Antibodies were validated for use in Luminex (see manufacturer's website):

17-plex ProcartaPlex Multiplex Immunoassay Invitrogen, lot number 178863000

IFNa, IFNb, IL-1b, IL10, IL12p70, IL13, IL15, IL2, IL22, IL23, IL4, IL6, IP10, MCP1, MIP1a, MIP1b and TNFa

Antibody was validated for direct immunofluorescence (see manufacturer's website):

Goat anti-human IgM (u-chain specific)-FITC antibody (Sigma-Aldrich; F5384)

All antibodies were validated for use in ELISA (see manufacturer's website)

Mouse anti human IgG alkaline phosphatase (Sigma-Aldric; A2064)

Antibodies used in experimental ELISA

Horseradish peroxidase (HRP-labelled anti human IgG1) (Sanquin, Amsterdam, the Netherlands)

HRP-labelled anti human IgG4 (Sanquin, Amsterdam, the Netherlands)

Alkaline phosphatase conjugated anti human IgG (Sigma, St Louis, MO, USA)

Biotinylated goat anti human IgE (Vector Laboratories, Burlingame, CA, USA)

Streptavidin HRP conjugate (Sanquin, Amsterdam, the Netherlands)

Rabbit anti-human IgE (Dako, Glostrup, Denmark)

\section{Animals and other organisms}

Policy information about studies involving animals; ARRIVE guidelines recommended for reporting animal research

Laboratory animals

Wild animals

Field-collected samples
The study did not involve laboratory animals. The S. mansoni cercariae used for infection were produced in laboratory-reared Biomphalaria glabrata snails according to the natural life cycle. The $\mathrm{S}$. mansoni lifecycle has been kept in water snails and hamsters within the LUMC institute since 1955. The hamsters are 9-10 weeks old during infection and 16-17 weeks old when used for perfusion. The snails are infected when they are approximately $0.4 \mathrm{~cm}$ and the first shed is when they are approximately $1 \mathrm{~cm}$. Appropriate institutional and national regulations and guidelines including animal welfare approval have been obtained for these activities.

The study did not involve wild animals.

The study did not involve samples collected from the field 


\section{Human research participants}

\section{Policy information about studies involving human research participants}

Population characteristics A total of 17 healthy volunteers between 18 and 45 years old, without a history of Schistosoma infection. Use of medication with possible interference with praziquantel, artesunate or lumefantrine was excluded.

The study groups were small (3-11 volunteers), showing differences in age (median 20, 23 and 30$)$ and in male/female ratio (0\%, $33 \%$, and $45 \%$ males).

Recruitment

Advertisements were placed in prominent places on the university and hospital campuses and other public places as well as on the internet and intranet site of the LUMC. Short presentations were held at student meetings or lectures. Furthermore, a Facebook page showing the advertisement text was designed to inform people about the trial. The study was be advertised on social media and local newspapers. This brief advertisement indicated a telephone number to call and an e-mail address for contact to request further information. It furthermore indicated a website which contained a form to request further information.

All volunteers with interest in participation in the study were advised to join one of the information meetings and received the study documents (the information sheet, the application form and the insurance text about the study. If they were still interested after the meeting or after reading the documents, an appointment for a screening visit was made. The screening visit was planned at least 72 hours after the subject received the information sheet and informed consent form.

Ethics oversight

The study was approved by the LUMC Institutional Medical Ethical Research Committee (IRB P16.111).

Note that full information on the approval of the study protocol must also be provided in the manuscript.

\section{Clinical data}

Policy information about clinical studies

All manuscripts should comply with the ICMJE guidelines for publication of clinical research and a completed CONSORT checklist must be included with all submissions.

Clinical trial registration

Study protocol

Data collection

Outcomes

\section{clinicaltrials.gov NCT02755324}

The full study protocol is available on request at the corresponding author

Recruitment was performed between 8 weeks to 3 days before exposure.

Volunteers were seen at the outpatient clinic weekly between week 0-12 and biweekly from week 12-24, and on week 52 after exposure.

Primary outcome:

o Frequency and magnitude of adverse events after controlled human Schistosoma mansoni infection with male cercariae. o The number of male cercariae at which 100\% volunteers show detectable Schistosoma mansoni circulating anodic antigen.

Exploratory outcome:

o Time to positive serum and urine CAA (Circulating Anodic Antigen) test;

o Comparison of the height of the peak serum CAA concentration in different dose groups.

o Humoral (antibody) responses directed against Sm antigens

o Cellular responses directed against $\mathrm{Sm}$ antigens

o Changes in microbiome after controlled human Schistosoma mansoni infection with male Sm cercariae.

o Inflammatory responses to $\mathrm{Sm}$ infection in sputum

\section{Flow Cytometry}

\section{Plots}

Confirm that:

\The axis labels state the marker and fluorochrome used (e.g. CD4-FITC).

X The axis scales are clearly visible. Include numbers along axes only for bottom left plot of group (a 'group' is an analysis of identical markers).

$\bigotimes$ All plots are contour plots with outliers or pseudocolor plots.

$\bigotimes$ A numerical value for number of cells or percentage (with statistics) is provided.

\section{Methodology}

Sample preparation
Between week 0 and 24 after exposure every 4 weeks human peripheral blood mononuclear cells (PBMC) were isolated from whole blood collected in heparin. Cells of two heparin tubes were diluted at least 1:2 with Hank's Balanced Salt Solution (HBSS, ThermoFisher, Waltham, MA, United States) at room temperature. $10 \mathrm{~mL}$ of ficoll at room temperature was added, followed by 
25 minutes centrifugation at $400 \mathrm{G}$ with low brake. Cells were collected and washed with HBSS, counted and frozen in $10 \%$ DMSO, 20\% FCS in RPMI Hepes (Invitrogen, Carlsbad, CA, USA), with $100 \mathrm{U} / \mathrm{ml}$ Penicillin, $100 \mathrm{ug} / \mathrm{ml}$ Streptomycin, Pyruvate 1 $\mathrm{mM}$ /Glutamate $2 \mathrm{mM}$ and 10\% FCS (Bodinco, Alkmaar, The Netherlands). Subsequently, PBMCs were thawed in RPMI Hepes, with $100 \mathrm{U} / \mathrm{ml}$ Penicillin, $100 \mathrm{ug} / \mathrm{ml}$ Streptomycin, Pyruvate $1 \mathrm{mM} / \mathrm{Glutamate} 2 \mathrm{mM}$ and $10 \% \mathrm{FCS}$ and rested overnight at $37^{\circ} \mathrm{C}$, $5 \%$ CO2. The cells were counted and transferred to a 96-wells plate round bottom (Corning NY, USA) with 500.000 cells/well. The cells were stimulated with AWA (50 ug/ml) for 24 hours. Staphylococcal enterotoxin B (SEB) (Sigma-Aldrich, Zwijndrecht, The Netherlands) $200 \mathrm{ng} / \mathrm{ml}$ was used as a positive control, and RPMI Hepes (Invitrogen), with $100 \mathrm{U} / \mathrm{ml}$ Penicillin, $100 \mathrm{ug} / \mathrm{ml}$ Streptomicin, Pyruvate $1 \mathrm{mM} /$ Glutamate $2 \mathrm{mM}$ and $20 \%$ FCS as a negative control. After 4 hours of incubation $5 \mathrm{mg} / \mathrm{ml}$ Brefeldin A (Sigma) was added to SEB stimulated wells, and after 20 hours to AWA and medium stimulated wells. After a total stimulation of 24 hours, the cells were stained with Aqua (Invitrogen) and fixed with 3,9\% Formaldehyde (Sigma). After fixation, the cells were stained with the following antibodies: CD3, CD4, IFNY, IL-2, Th2-cytokines (IL-4/IL-5/IL-13), TNF, IL-10 (table 1). Human FC block was used to avoid non-specific interactions. The left over, stimulated, aqua stained and fixed cells were frozen in 10\% DMSO, in RPMI Hepes, with $100 \mathrm{U} / \mathrm{ml}$ Penicillin, $100 \mathrm{ug} / \mathrm{ml}$ Streptomycin, Pyruvate $1 \mathrm{mM} /$ Glutamate $2 \mathrm{mM}$ and 20\% FCS and stored by $-80^{\circ} \mathrm{C}$. The cells were thawed by $37^{\circ} \mathrm{C}$ and stained with the following antibodies: CD3, CD4, CD25, CD127 and FOXP3 (table 1). Human FC block was used to avoid non-specific interactions.

Instrument

Software

Cell population abundance

Gating strategy

\section{FACSCanto II (BD Biosciences Franklin Lakes, NJ, USA)}

The data was analyzed with FlowJo 10.5 software for MAC OS.

For each sample, 500.000 cells per well were stimulated 24 hours with medium (as negative control), adult worm antigen (AWA) or Staphylococcal Enterotoxin B (SEB, as positive activation control) (100ul). 24 hours after AWA-stimulation, fixing and staining an average of 35.302 single cells were left. On average of 90,8\% (31.791) of these were alive. Of this alive population on average $47,3 \%(15.530)$ CD4+ cells were gated.

The FMO controls as well as the medium and SEB control were used to set the gating. The following order of gating was used for the cytokines: Lymphocytes, Single cells, Life/dead cells (Aqua AmCyan), CD4+ cells (PerCP-ef710), different cytokines: IL2 (FITC), IL-4+IL-5+IL-13 as TH2 respons (PE), TNFa (PE-Cy7), IL-10 (APC), IFNg (BV421).

The following order of gating was used for the FOXP3+CD25+CD4+ cells: Lymphocytes, Single cells, Life/dead cells (Aqua AmCyan), CD4+ cells (PerCP-ef710), CD25+ cells (BV421), FOXP3+ cells (APC). After the gating for the controls was optimized, the same gating strategy was used for all the samples. Flowjo 10.5 was used for the gating.

The gating was placed with help of fluorescence minus one (FMO's) controls, the medium as a negative control and SEB as a positive control.

Х Tick this box to confirm that a figure exemplifying the gating strategy is provided in the Supplementary Information. 